\title{
Selective Restoration of Pomc Expression in Glutamatergic POMC Neurons: Evidence for a Dynamic Hypothalamic Neurotransmitter Network
}

\author{
(1) Graham L. Jones, ${ }^{1,2}$ Gábor Wittmann, ${ }^{3}$ Eva B. Yokosawa, ${ }^{2}$ Hui $\mathrm{Yu}^{2}{ }^{2}$ Aaron J. Mercer, ${ }^{2}$ Ronald M. \\ Lechan, ${ }^{3}$ and ${ }^{-M a l c o l m ~ J . ~ L o w ~}{ }^{2}$
}

https://doi.org/10.1523/ENEURO.0400-18.2019

\begin{abstract}
${ }^{1}$ Neuroscience Graduate Program, ${ }^{2}$ Department of Molecular and Integrative Physiology, University of Michigan Medical School, Ann Arbor, MI 48105, and ${ }^{3}$ Department of Medicine, Division of Endocrinology, Diabetes and Metabolism, Tufts Medical Center, Boston, MA 02111
\end{abstract}

\begin{abstract}
Hypothalamic POMC deficiency leads to obesity and metabolic deficiencies, largely due to the loss of melanocortin peptides. However, POMC neurons in the arcuate nucleus (ARC) are comprised of glutamatergic and GABAergic subpopulations. The developmental program, relative proportion and function of these two subpopulations are unresolved. To test whether glutamatergic POMC neurons serve a distinct role in maintaining energy homeostasis, we activated Pomc expression Cre- dependently in Vglut2-expressing neurons of mice with conditionally silenced Pomc alleles. The Vglut2-Pomc restored mice had normal ARC Pomc mRNA levels, POMC immunoreactivity, as well as body weight and body composition at age 12 weeks. Unexpectedly, the cumulative total of Vglut2 $^{+}$glutamatergic- and Gad67 ${ }^{+}$GABAergic-Pomc neurons detected by in situ hybridization (ISH) exceeded $100 \%$ in both Vglut2- Pomc restored and control mice, indicating that a subpopulation of Pomc neurons must express both neuronal markers. Consistent with this hypothesis, triple ISH of C57BL/6J hypothalami revealed that $35 \%$ of ARC Pomc neurons were selectively Gad $67^{+}, 21 \%$ were selectively Vglut2 ${ }^{+}$, and $38 \%$ expressed both Gad67 and Vglut2. The single Gad $67^{+}$and Vglut2 ${ }^{+}$Pomc neurons were most prevalent in the rostral ARC, while the Vglut2/Gad67 $7^{+}$dual-phenotype cells predominated in the caudal ARC. A lineage trace using Ai9-tdTomato reporter mice to label fluorescently all Vglut2-expressing neurons showed equal numbers of tdTomato $^{+}$and tdTomato ${ }^{-}$POMC immunoreactive neurons. Together, these data suggest that POMC neurons exhibit developmental plasticity in their expression of glutamatergic and GABAergic markers, enabling reestablishment of normal energy homeostasis in the Vglut2-Pomc restored mice.
\end{abstract}

Key words: GABA; glutamate; neurotransmitter flexibility; obesity; POMC

\section{Significance Statement}

Melanocortin peptides secreted from hypothalamic POMC neurons are anorexigenic and play a critical role in preventing obesity. However, POMC neurons are heterogeneous in their synaptic release of the neurotransmitters glutamate and GABA. We used a conditional gene expression approach to test the hypothesis that these two subsets of POMC neurons influence distinct neural circuits controlling energy homeostasis. Unexpectedly, we found that Vglut2-IRES-Cre dependent Pomc restoration on a hypothalamic Pomc null background was sufficient to prevent obesity. A series of double-label and triple-label in situ hybridization (ISH) experiments showed that 38\% of POMC neurons express both S/c17a6 and Gad67, markers of glutamatergic and GABAergic neurons, respectively. Together, these data suggest a previously unreported developmental plasticity in the neurotransmitter phenotype of POMC neurons. 


\section{Introduction}

POMC-derived peptides are critical in maintaining energy balance and body composition, as well as in regulating feeding behavior. Neuronal Pomc deficiency leads to morbid obesity, hyperphagia, hypolocomotion, and metabolic abnormalities (Bumaschny et al., 2012; Lam et al., 2015b; Chhabra et al., 2016a,b). It was identified previously that POMC neurons are comprised of both GABAergic and glutamatergic cells (Hentges et al., 2004, 2009; Mercer et al., 2013). However, little is known about the functional impact or genetic programs of these neuronal subclasses. Furthermore, there is not a consensus on the relative proportions of each POMC neuron subtype, due to differences in methodologies used to classify the cells (Hentges et al., 2009; Vong et al., 2011; Dicken et al., 2012; Wittmann et al., 2013; Dennison et al., 2016).

Many studies have revealed plasticity in neurotransmitter identity or in neurotransmitter co-release. These phenomena are evident during development or in response to environmental stimuli, and span diverse cell groups and neurotransmitter types (Walker et al., 2001; Gutiérrez, 2002, 2003; Gutiérrez et al., 2003; Kao et al., 2004; Ottem et al., 2004; Gillespie et al., 2005; Gómez-Lira et al., 2005; Zander et al., 2010; Mestikawy et al., 2011; Dulcis et al., 2013; Root et al., 2014; Spitzer, 2015, 2017; Meng et al., 2018). Work by Dennison et al. (2016) uncovered a postnatal shift in the proportions of Vglut2 $^{+}$and $\mathrm{Gad}_{67}{ }^{+}$ POMC neurons, where the Vglut2-Pomc overlap was the highest $(\sim 40 \%)$ immediately after birth, but was reduced by 4 -fold $(\sim 10 \%)$ when the animals had matured to eight weeks old. The opposite temporal pattern was observed with Gad67-Pomc overlap. However, the mechanism of this shift in proportionality is unclear. One possibility is that POMC neurons are glutamatergic early in hypothalamic development and then transdifferentiate to a GABAergic phenotype in postnatal life. Alternatively, there

Received October 16, 2018; accepted February 17, 2019; First published March 4, 2019

The authors declare no competing financial interests.

Author contributions: G.L.J., G.W., R.M.L., and M.J.L. designed research; G.L.J., G.W., E.B.Y., and A.J.M. performed research; G.L.J., G.W., and H.Y. analyzed data; G.L.J., G.W., and M.J.L. wrote the paper.

This work was supported by National Institutes of Health Grants R01DK066604 (to M.J.L.), the Systems and Integrative Biology Training Grant T32-GM008322 (to G.L.J.), the Early Stage Neurosciences Training Grant T32-NS076401 (to G.L.J.), the Ruth L. Kirschstein National Research Service Award (NRSA) Individual Postdoctoral Fellowship F32-DK098051 (to A.J.M.), and a grant from the Dr. Gerald J. and Dorothy R. Friedman New York Foundation for Medical Research (R.M.L.) and utilized services of the Imaging Core supported by the Michigan Diabetes Research Center (P30-DK020572) and the Metabolism and Behavior Core supported by the Michigan Mouse Metabolic Phenotyping Center (U2C-DK110768), the Michigan Diabetes Research Center (P30-DK020572), and the Michigan Nutrition Obesity Center (P30-DK089503).

We thank the members of the Low and Lechan labs for scientific feedback, specifically, Jessica Adams for the GPCR primers and guidance, and the Metabolism and Behavior Core for conducting the NMR measurements.

Correspondence should be addressed to Malcolm J. Low at mjlow@umich.edu. https://doi.org/10.1523/ENEURO.0400-18.2019

Copyright (C) 2019 Jones et al.

This is an open-access article distributed under the terms of the Creative Commons Attribution 4.0 International license, which permits unrestricted use, distribution and reproduction in any medium provided that the original work is properly attributed. may be a selective increase in the absolute number of de novo GABAergic POMC neurons that arise postnatally. There are also reports of some POMC neurons expressing both Vglut2 ${ }^{+}$and Gad67 ${ }^{+}$(Jarvie and Hentges, 2012), indicating the possibility of a shift between neurotransmitter phenotypes or the potential that a subset of POMC neurons can synaptically release both glutamate and GABA. Recent work from Stincic et al. (2018) also indicates that POMC neurons can locally regulate the function of neuropeptide Y/agouti-related peptide neurons in the arcuate nucleus (ARC) via glutamatergic and $\beta$-endorphin input, expanding our functional understanding of melanocortin circuitry. Additionally, there are data indicating a partial dissociation between peptidergic and fast neurotransmitter synaptic terminals from POMC neuron projections at sites throughout the brain (Mercer et al., 2014), further complicating the interpretation of the specific functions of these neurons.

This study was conceived initially to test the hypothesis that glutamatergic and GABAergic POMC neurons serve distinct and dissociable roles in overall POMC neuron function related to the maintenance of energy homeostasis. We chose to investigate the impact of selectively restoring Pomc function in the developing hypothalamus from a conditionally silent allele (Fneo $\Delta 2$ mice) using a Vglut2-IRES-Cre knock-in mouse model (Vong et al., 2011) and then determine how restoration of Pomc expression only in the glutamatergic subpopulation of POMC neurons shapes hypothalamic POMC neural circuitry and impacts energy balance in the obesitydestined mice. Additionally, we sought to capture the overlap between glutamate-associated neurons and hypothalamic POMC expression. We also used Vglut2-Cre-driven reporter expression to perform a lineage trace of all cells that have expressed Vglut2 at some point in their existence to compare with POMC immunoreactivity. Finally, triple-label in situ hybridization (ISH) was performed on wild-type tissue to establish the degree of overlap between Pomc, Vglut2, and Gad67 gene expression in adult mice.

\section{Materials and Methods}

\section{Animal care}

All animal procedures were performed in accordance with the University of Michigan IACUC regulations. Mice were housed under a 12/12 $\mathrm{h}$ light/dark photoperiod at constant temperature of $22^{\circ} \mathrm{C}$ in ventilated cages with ad libitum access to water and chow (5LOD; LabDiet containing $28.5 \mathrm{kcal} \%$ protein, $13.5 \mathrm{kcal} \%$ fat, and $58.0 \mathrm{kcal} \%$ carbohydrates).

\section{Mouse strains and breeding strategy}

Ai9 tdTomato reporter mice (Allen Institute, The Jackson Laboratory; Gt(ROSA)26Sor ${ }^{\text {tm9(CAG-tdTomato)Hze })}$ were crossed to Vglut2-ires-Cre/ ${ }^{+}$mice (The Jackson Laboratory; Slc17a6 ${ }^{\text {tm2(cre)Lowl } / J ; ~ V o n g ~ e t ~ a l ., ~ 2011) ~ t o ~ g e n e r a t e ~}$ Vglut2-tdTomato compound mice for a developmental lineage trace of all neurons that have expressed the gene encoding the vesicular glutamate transporter Vglut2 at some point in their existence. Male (M) and female (F) mice were used in all experiments.

ArcPomc $^{+/}$(ARC specific Cre-reversible Pomc KO or Fneo $\Delta 2$ ) mice (Bumaschny et al., 2012; Lam et al., 2015a; 
Chhabra et al., 2016a,b) were crossed to Vglut2 ${ }^{\mathrm{Cre} /+}$ mice to obtain compound heterozygous Vglut2 ${ }^{\mathrm{Cre} /+}$; arcPomc $^{+/-}$mice. Those mice were back crossed to $\mathrm{arcPomc}^{+/-}$mice to yield the control and experimental groups for $\mathrm{POMC}$ restoration and ISH studies. These three groups were: Vglut2 ${ }^{\mathrm{Cre} /+} ; \mathrm{Pomc}^{+/+}$(control), Vglut2 ${ }^{+/+}$; arcPomc $^{-/-}$(FN $\Delta 2$ ) and Vglut2 ${ }^{\mathrm{Cre} /+}$; $\operatorname{arcPomc}^{-/-}$ (restored). FN $\Delta 2$ animals have a floxed-neomycin cassette inserted between neural Pomc enhancer 1 (nPE1) and the deleted neural Pomc enhancer 2 ( $\Delta$ nPE2) locus, which prevents the transcription of Pomc in neurons, while leaving pituitary transcription intact. After Cre-mediated excision of the floxedneomycin cassette, neuronal Pomc transcription is restored from the functional nPE1 enhancer.

\section{Growth curves, body composition, and tissue collection}

Mice were weighed weekly from ages 3-12 weeks. Body composition was assessed by nuclear magnetic resonance (NMR; Minispec LF90 II, Bruker Optics) at age 12 weeks. Following NMR, a cohort of animals was killed by decapitation; gonadal and inguinal fat pads were collected and weighed, and bilateral $2-\mathrm{mm}^{3}$ tissue blocks were collected from the medial-basal hypothalamus (coordinates from bregma; A-P: -1 to $-3 \mathrm{~mm}, \mathrm{M}-\mathrm{L}$ : \pm 0 to 1 $\mathrm{mm}$, and $\mathrm{D}-\mathrm{V}$ : 0 to $1 \mathrm{~mm}$ from ventral surface) and the dorsal striatum (coordinates from bregma; $A-P$ : +1 to -1 $\mathrm{mm}, \mathrm{M}-\mathrm{L}: \pm 1$ to $2 \mathrm{~mm}$, and $\mathrm{D}-\mathrm{V}$ : -2.5 to $-3.5 \mathrm{~mm}$ from dorsal surface) for use in genomic DNA PCR and qRTPCR. The brains used in the ISH studies were collected at age 9-13 weeks, and fresh tissue was flash frozen using isopentane (2-methyl butane) cooled on dry-ice. Tissue used for immunohistochemistry (IHC) was collected from 12- to 13-week-old mice, anesthetized with an overdose of $2 \%$ tribromoethanol (Avertin; $400 \mathrm{mg} / \mathrm{kg}$, i.p.) and perfused transcardially with PBS ( $\mathrm{pH} 7.4)$, followed by $4 \%$ paraformaldehyde (PFA; Sigma-Aldrich; catalog \#158127) dissolved in PBS (pH 7.4). Brains were post-fixed overnight in $4 \%$ PFA at $4^{\circ} \mathrm{C}$, and then cryoprotected with $30 \%$ sucrose (ThermoFisher Scientific; catalog \#BP220) in PBS (pH 7.4).

\section{PCR verification of Cre-mediated genomic DNA recombination in restored mice}

Genomic DNA samples were extracted from one of the bilateral 2- $\mathrm{mm}^{3}$ blocks of fresh brain tissue described above. The samples were then analyzed by PCR using primers designed to detect the presence of the floxed neomycin cassette and the recombined DNA sequence following Cre-mediated excision of the neo cassette (forward1: TACTTGGGCCTCAGGGTACTGAAA, $0.67 \mu \mathrm{M}$; forward2: TGGGGCTCGACTAGAGGAT, $0.67 \mu \mathrm{M}$; reverse: CCCATCCAGCTACAGCTGT, $0.67 \mu \mathrm{M}) ; 25-\mu$ l PCRs were set up using $5 \times$ Green GoTaq reaction buffer (Promega; catalog \#M7123), the aforementioned primers, the extracted DNA, BioReady rTaq DNA Polymerase (Bulldog Bio, catalog \#BSA12L050), and nuclease-free water. The reactions were run using a touchdown protocol on a Peltier Thermal Cycler (MJ Research; PTC-100). The reaction conditions started with a 4-min denaturing step at $94^{\circ} \mathrm{C}$, followed by a 16-cycle touchdown, where each cycle starts with a 1 -min denaturing step at $94^{\circ} \mathrm{C}$, fol- lowed by a 1-min annealing step starting at $67^{\circ} \mathrm{C}$ and decreasing by $1^{\circ} \mathrm{C}$ each cycle, and a 1-min extension at $72^{\circ} \mathrm{C}$. Following the 16 touchdown cycles were 16 additional cycles structured in the same way, except that the annealing temperature was constant at $52^{\circ} \mathrm{C}$. Thermal cycling terminated with a 10 -min extension step at $72^{\circ} \mathrm{C}$, followed by holding at $4^{\circ} \mathrm{C}$. PCR products were then run on a $2 \%$ agarose gel in TBE buffer. Gel images were processed in ImageJ to measure relative band intensity of the two PCR products. Recombined band intensities were quantified from neural tissue collected from the restored mice as follows: (recombined (287 bp) product - gel background)/(non-recombined (180 bp) product - background). Quantified band intensities were compared between the medial-basal hypothalamus and the dorsal striatum of restored mice, using a paired $t$ test.

\section{RNA extraction, cDNA synthesis, and qRT-PCR}

RNA was extracted from one of the bilateral $2-\mathrm{mm}^{3}$ blocks of the medial-basal hypothalamus and analyzed for Pomc transcript expression using qRT-PCR. RNA was extracted from hypothalamic samples homogenized by trituration in $50 \mu$ l of TRIzol reagent (ThermoFisher Scientific, Ambion, Life Technologies; catalog \#15596). Following extraction, the RNA samples were treated with a TURBO DNA-free kit (ThermoFisher Scientific, Ambion, Life Technologies; catalog \#AM1907) to remove residual genomic DNA. Then, 500 ng of each RNA sample was converted to a 20- $\mu$ l cDNA library using the GoScript Reverse Transcription System (Promega; catalog \#A5000), after which the libraries were diluted 1:4 in nuclease-free water.

A total of $20 \mu \mathrm{l}$ qPCRs were set up using $2 \times$ SYBR Green PCR Master Mix (ThermoFisher Scientific, Applied Biosystems; catalog \#4309155), and Pomc-transcript or Ppia-transcript primers, with $2 \mu \mathrm{l}$ of diluted cDNA, and nuclease-free water. The Pomc primers were designed to span exons 2 and 3 of splice variant 1 (forward: GAGCTGGTGCCTGGAGAG, 300 nM; reverse: T1TCAGTCAGGGGCTGTTC, $300 \mathrm{nM})$. The Ppia primers were designed to span exons 1 and 3 of all splice variants (forward: CACCGTGTTCTTCGACATCA, 300 nM; reverse: CAGTGCTCAGAGCTCGAAAGT, $300 \mathrm{nM})$. The reactions were performed in duplicate and loaded onto a MicroAmp Fast Optical 96-well reaction plate (ThermoFisher Scientific, Applied Biosystems; catalog \#4346906) and run on a StepOnePlus Real-Time PCR System (ThermoFisher Scientific, Applied Biosystems; catalog \#4376600). The reaction conditions started with a 10-min denaturing step at $95^{\circ} \mathrm{C}$, followed by 40 cycles of a two-step PCR protocol with a 15 -s $95^{\circ} \mathrm{C}$ denaturing step and a 1 -min $60^{\circ} \mathrm{C}$ annealing step.

$\mathrm{C}_{\mathrm{T}}$ values were determined by manually setting the threshold at 1, which was in the middle of the exponential phase of amplification for each sample. Baseline readings were automatically assessed by the StepOne Software (ThermoFisher Scientific, Applied Biosystems). Standard curves for each transcript were established by pooling equal amounts of cDNA from all control samples and making serial dilutions $(1: 1,1: 4,1: 16,1: 64,1: 256$, and 1:1024). The percent dilution was $\log _{10}$ transformed (e.g., 
$1: 4=25 \%$ and $\left.\log _{10}(25)=1.398 \%\right)$ and plotted against its respective $\mathrm{C}_{\mathrm{T}}$ value. The slope and $\mathrm{y}$-intercept of the line formed between all of the dilutions were used to evaluate the relative $\log _{10}$ copy number for each sample (i.e., $\log _{10}$ copy number $=$ [sample $\mathrm{C}_{\mathrm{T}}-\mathrm{Y}$ intercept]/ slope), which was then linearized. For each sample, the Pomc and Ppia linear copy numbers were averaged across duplicates and the Pomc average was divided by the Ppia average, to yield a normalized Pomc expression value. The efficiency of amplification was $>90 \%$. Pomc expression values for each sample were then standardized to the group average of the control animals to generate a relative quantification of Pomc transcript expression. Due to the number of samples, male and female samples were run independently on separate plates with the same standard dilutions, and then normalized within sex.

\section{IHC}

Sucrose-equilibrated brains, preparation described above, were cryosectioned at $30 \mu \mathrm{m}$ and collected in triplicate with a freezing stage sliding microtome (Leica Biosystems; SM 2010R) into PBS. The sections were then incubated with antisera to POMC [1:1000 (control and restored mice) or 1:10,000 (Vglut2-tdTomato animals); Phoenix; rabbit; H-029-30]. Following triplicate washes, one set of the sections from control and restored mice was incubated with a biotinylated goat anti-rabbit secondary antisera (1: 500 , Vector Labs; catalog \#BA-1000 ROS23) followed by treatment with a Vectastain ABC HRP kit (Vector Labs; catalog \#PK-4000) and development of a colorimetric stain with diaminobenzidine $\left(250 \mu \mathrm{g} / \mathrm{ml}\right.$ in TBS with $\left.0.1 \% \mathrm{H}_{2} \mathrm{O}_{2}\right)$. A second set of sections from control and restored mice was incubated with Alexa Fluor 568 (A568) goat anti-rabbit secondary antibody (1:500; ThermoFisher Scientific; catalog \#A11036). Vglut2-tdTomato brain sections were incubated with Alexa Fluor 488 (A488) goat anti-rabbit secondary antibody (1:500; ThermoFisher Scientific; catalog \#A-11034).

\section{IHC image analysis}

Images from the DAB-treated IHC tissue sections were acquired with a $6.3 \times / 0.20160 \mathrm{NPL}$ Fluotar objective on a Leitz Dialux 22 microscope with a Leica DFC280 camera (Leitz/Leica). Fluorescent images used for the Pomcrestoration and tdTomato-POMC overlap counts were taken with a $10 \times$ objective on a Nikon Eclipse 90i digital upright microscope with a Photometrics CoolSNAP HQ2 CCD Camera. The images were acquired using a 1-s exposure for the POMC signal and a 50-ms exposure time for the tdTomato signal. Resulting tdTomato pictures were then double-processed with ImageJ (http://imagej.nih.gov/ij/), by adjusting the brightness and contrast, to account for different background fluorescence levels in the GABA-rich basomedial ARC and in the glutamate-rich lateral ARC. The representative image shown was acquired using a Nikon A1 confocal microscope.

\section{Hybridization probes}

Riboprobes for ISH were generated from cDNA sequences as follows (NCBI GenBank accession numbers in parenthesis): mouse Pomc, bases 502-1008 (short probe) or bases 1-1008 (long probe; NM_001278584.1); Vglut2, bases 1762-2390 (NM 080853.3); and Gad67, bases 317-892 (NM_008077.4). The Vglut2 and Gad67 plasmid templates are a gift from Dr. Erik Hrabovszky (Institute of Experimental Medicine, Budapest), the long Pomc template was synthesized by Genscript. For dual-label ISH, the short Pomc probe was labeled with digoxigenin-11UTP (Roche Applied Sciences), and the Vglut2 and Gad67 probes with $\left[{ }^{35} \mathrm{~S}\right]$-uridine $5^{\prime}$ - $(\alpha$-thio) triphosphate (PerkinElmer). For triple-label ISH, the long Pomc probe was labeled with fluorescein-12-UTP (Roche), the Gad67 probe with digoxigenin-11-UTP, and the Vglut2 probe $\left[{ }^{35} \mathrm{~S}\right]$-uridine $5^{5}$ ( $\alpha$-thio) triphosphate.

\section{Dual-label ISH}

Sixteen-micrometer coronal sections were cut through the rostrocaudal extent of the hypothalamic ARC using a Leica CM3050 S cryostat, thaw-mounted on Fisherbrand Superfrost Plus Microscope Slides (ThermoFisher Scientific; catalog \#12-550-15), air-dried and stored at $-80^{\circ} \mathrm{C}$. The mounted sections were fixed with 4\% PFA in $0.1 \mathrm{M}$ phosphate buffer $(\mathrm{pH}$ 7.4) for 20 min, rinsed in PBS for 5 min, acetylated with $0.25 \%$ acetic anhydride in $0.1 \mathrm{M}$ triethanolamine for $10 \mathrm{~min}$, treated with ascending ethanol series and chloroform (10 min), partially rehydrated in $95 \%$ ethanol, and then processed for hybridization. Two adjacent series of sections, each containing every 7 th section, were hybridized with the mixture of the digoxigenin-labeled short Pomc riboprobe and the $\left[{ }^{35} \mathrm{~S}\right]-$ labeled riboprobe for either Vglut2 or Gad67 (diluted to $50,000 \mathrm{cpm} / \mu \mathrm{l}$ ) overnight at $56^{\circ} \mathrm{C}$ in a humidified chamber. The Pomc probe was detected with peroxidaseconjugated anti-digoxigenin antibody (diluted 1:100 in 1\% blocking reagent, Roche), amplified using the TSA Biotin Tyramide system (PerkinElmer) for $30 \mathrm{~min}$ and labeled with Alexa Fluor 488-conjugated streptavidin (diluted 1:500 in 1\% blocking reagent, Life Technologies) for $2 \mathrm{~h}$. To detect the radiolabeled Vglut2 or Gad67 probe, sections were then rinsed in PBS, dehydrated, air-dried, and coated with Kodak NTB autoradiography emulsion (Carestream Health Inc.). The autoradiograms were developed using Kodak D19 developer after $7 \mathrm{~d}$ (Gad67) or $12 \mathrm{~d}$ (Vglut2) of exposure.

\section{Triple-label ISH}

Triple-label ISH was performed on serial hypothalamic sections of three male and three female wild-type C57BL/6J mice, euthanized on postnatal day 65 by decapitation under deep ketamine/xylazine anesthesia. Tissue collection and processing was identical to the duallabel ISH procedure. Sections were hybridized with the mix of the fluorescein-labeled long Pomc, digoxigeninlabeled Gad67, and $\left.{ }^{35} \mathrm{~S}\right]$-labeled Vglut2 riboprobes. Following hybridization, sections were first incubated in the peroxidase-conjugated sheep anti-digoxigenin antibody, and the signal was amplified with the TSA Plus DIG kit (catalog \#NEL748E001KT, PerkinElmer) for $30 \mathrm{~min}$, using the DIG amplification reagent at 1:500 dilution in $0.05 \mathrm{M}$ Tris ( $\mathrm{pH}$ 7.6) containing $0.01 \% \mathrm{H}_{2} \mathrm{O}_{2}$. Sections were then incubated in a rabbit monoclonal anti-digoxigenin antibody (ThermoFisher, catalog $\# 700772$; at $1-\mu \mathrm{g} / \mathrm{ml}$ concentration) for $3 \mathrm{~h}$, in the presence of $2 \%$ sodium azide to 
inactivate peroxidase activity. Sections were thoroughly washed in PBS, and incubated overnight in peroxidaseconjugated sheep anti-fluorescein antibody (Roche, catalog \#11426346910; diluted 1:100 in 1\% blocking reagent). Signal amplification was applied for $30 \mathrm{~min}$, using the TSA Plus Biotin kit (PerkinElmer) with the TSA Plus biotin reagent diluted $1: 300$ in $0.05 \mathrm{M}$ Tris and $0.01 \%$ $\mathrm{H}_{2} \mathrm{O}_{2}$. The biotin deposits and the anti-digoxigeninantibody were detected with Alexa Fluor 488-conjugated streptavidin and Alexa Fluor 594-conjugated anti-rabbit IgG (Jackson ImmunoResearch; 1:200), respectively. The sections were then dehydrated, dipped in Kodak NTB autoradiography emulsion, and developed after $10 \mathrm{~d}$ as described above.

\section{ISH image analysis}

Fluorescent signals and darkfield emulsion autoradiography images of the same field were captured with the $10 \times$ objective of a Zeiss Axioplan 2 microscope (Carl Zeiss) equipped with a RT SPOT digital camera (Diagnostic Instruments). Every 14th section (five sections per mouse), covering the rostro-caudal extent of the Arc was used to count the number of Pomc, dual-labeled PomcVglut2 and Pomc-Gad67, and triple-labeled PomcVglut2-Gad67 neurons. To be considered specifically labeled with the radioactive Vglut2 or Gad67 probes, Pomc neurons had to exhibit at least a 5-fold higher silver grain density than over background regions. This was confirmed by ImageJ for each Pomc neuron with lighter silver grain label. For publication images, the green fluorescence of Alexa Fluor 488 (Pomc) was pseudocolored to either red or blue as noted in the two relevant figure legends to better visualize colocalization with the silver grain autoradiography signal.

\section{Data analysis}

All statistical testing was performed using GraphPad Prism 7.04 for Windows (GraphPad Software). Datasets with an $n \geq 8$ were tested for normality using the D'Agostino and Pearson normality assessment, while groups with $n<8$, were assessed with the Shapiro-Wilk normality test. All sample sizes, mean \pm SEM, and measured units are located in the descriptive statistics table (Table 1). The data structures, specific statistical tests used, and numerical results are located in statistical tests table (Table 2). Both Tables 1, 2 are organized and labeled according to the respective figure panels representing the data, or when appropriate, annotated as not shown.

\section{Results}

A lineage trace of Vglut2-tdTomato neurons revealed that approximately half of all immunoreactive POMC neurons were colabeled with tdTomato

Because of differing reports for the percentage overlap of Vglut2 expression in POMC neurons (Hentges et al., 2009; Vong et al., 2011; Dicken et al., 2012; Wittmann et al., 2013; Dennison et al., 2016), we initially performed a lineage trace by crossing Vglut2-IRES-Cre mice with floxed tdTomato mice. In contrast to data obtained by the laboratory that originally generated the Vglut2-IRES-Cre strain, which showed only a $10 \%$ overlap of their lineage trace with POMC neurons (Vong et al., 2011), we found that there was a nearly even split in the number of POMC neurons that were co-labeled or unlabeled with the Vglut2-tdTomato reporter (Fig. 1A-H). There was no effect of the rostral-caudal position in the ARC on the overlap counts (Fig. 1/). The reason for this large discrepancy in the fraction of glutamatergic POMC neurons is unclear, but there were technical differences in the identification method for POMC neurons. The previous group performed their lineage trace using a second transgenic reporter strain, POMC-hrGFP, and then quantified the coexpression of tdTomato and the surrogate marker hrGFP. We chose to quantify the overlap of tdTomato with $\mathrm{POMC}$ neurons using the immunohistochemical detection of POMC itself.

\section{Vglut2-Cre-mediated activation of Pomc expression in the hypothalamus of restored mice normalized body weight and composition, and POMC immunoreactivity}

The specificity of Vglut2-ires-Cre mediated DNA recombination was assessed using PCR analysis of genomic DNA extracted from the relatively glutamatergic neuron-rich medial-basal hypothalamus and the relatively GABAergic neuron-rich dorsal striatum from restored mice. The three primer PCR design amplifies a 180-bp band from the intact FN $\Delta 2$ allele and a 287-bp band from the same allele after Cre-mediated recombination (Fig. $2 A, B$ ). No PCR product is generated from control DNA because the reverse primer cannot hybridize to the intact nPE2 enhancer. The degree of recombination was significantly higher in hypothalamic samples compared to striatal samples and there was no evidence of recombination in the brains of FN $\Delta 2$ mice lacking the Vglut2-IRES-Cre allele (Fig. 2C).

Weekly body weights showed identical growth curves between restored and control mice. Both groups diverged significantly from $\mathrm{FN} \Delta 2$ mice by age five (female) or six (male) weeks (Fig. $3 A, B$; only pairwise comparisons from one week prior to divergence to one week following are included in Table 2). Body composition measurements by NMR confirmed that these differences in body weight were due to excess fat mass in $\mathrm{FN} \Delta 2$ mice compared to control and restored mice, with no differences in lean mass across all groups (Fig. 3C,D). Furthermore, weights of the gonadal and inguinal fat pads showed that there were no depot-specific differences between restored and control animals, while both fat pads in each group were substantially smaller than those from obese FN $\Delta 2$ mice (Fig. 3E,F).

$\mathrm{IHC}$ for POMC was performed on neural tissue from control and restored mice. Three sections from each mouse taken between A-P coordinates from -1.5 to -1.9 $\mathrm{mm}$ posterior to bregma were included for analysis. There was no difference in either fluorescent (Fig. $4 A, B$ ) or DAB treated (Fig. $4 C, D$ ) cell counts between restored and control mice. However, there was an overall difference between labeling methods. DAB-treated sections displayed significantly higher cell counts than fluorescently-labeled sections (Fig. 4E). 
Table 1. Descriptive statistics

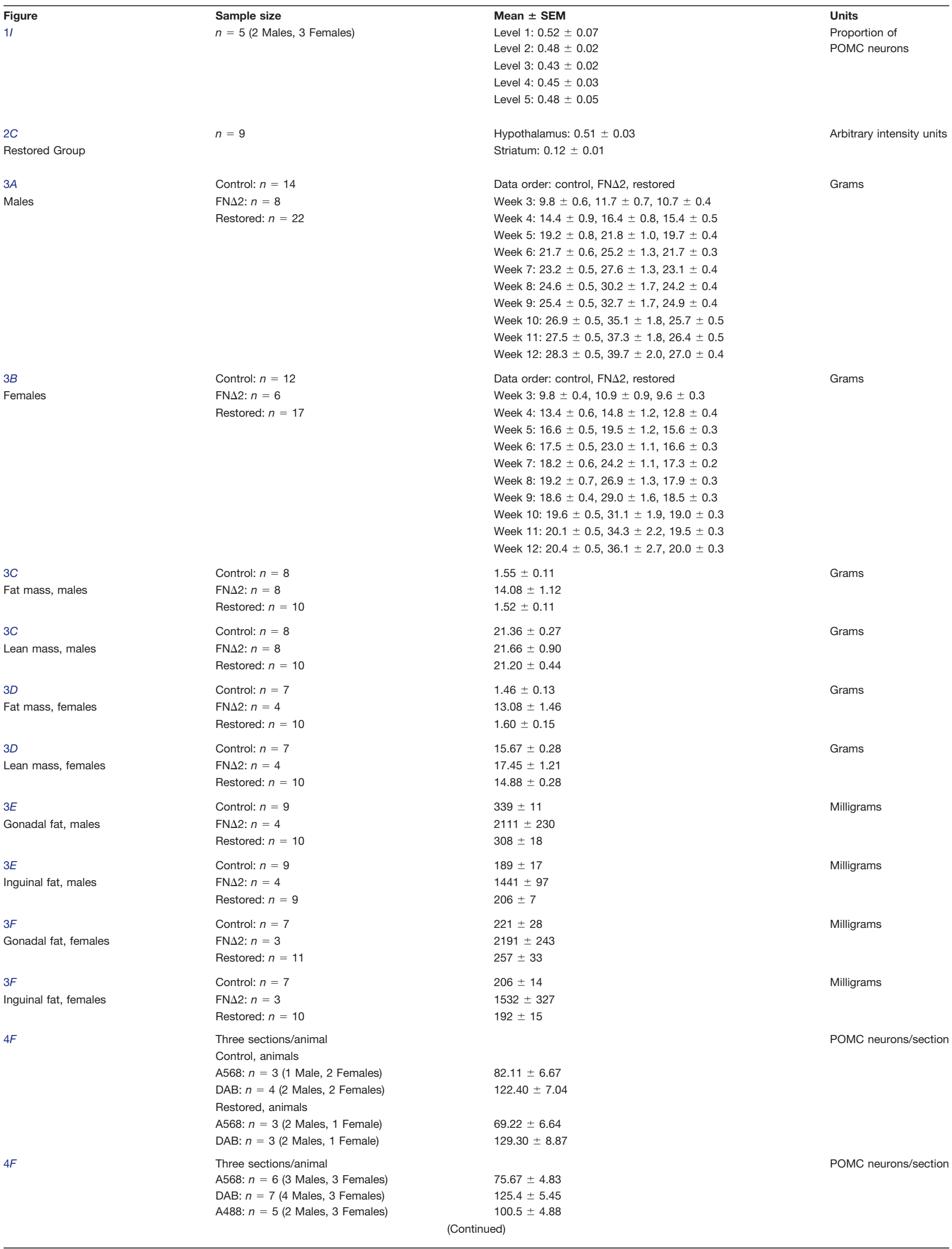


Table 1. Continued

\begin{tabular}{|c|c|c|c|}
\hline Figure & Sample size & Mean \pm SEM & Units \\
\hline 5 (data not shown) & Control: $n=6$ (4 Males, 2 Females) & $606.30 \pm 7.28$ & Number of Pomc neurons \\
\hline number of Pomc neurons & Restored: $n=9$ (6 Males, 3 Females) & $407.10 \pm 14.19$ & \\
\hline \multirow[t]{4}{*}{$5 E$} & Control: $n=6$ (4 Males, 2 Females) & Vglut2 $^{+}: 55.77 \pm 1.86$ & $\%$ of $P$ omc neurons \\
\hline & & 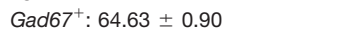 & \\
\hline & Restored: $n=8$ (5 Males, 3 Females) & Vglut2 $^{+}: 65.80 \pm 1.95$ & \\
\hline & & Gad $67^{+}: 60.59 \pm 1.29$ & \\
\hline $5 E$ (data not shown) & Control: $n=6$ (4 Males, 2 Females) & $-8.87 \pm 2.21$ & $\%$ Vglut2 $^{+}, \%$ Gad $67^{+}$ \\
\hline Difference & Restored: $n=8$ (5 Males, 3 Females) & $5.21 \pm 2.14$ & \\
\hline \multirow[t]{4}{*}{$5 F$} & Control: $n=6$ (4 Males, 2 Females) & Vglut2 $^{+}: 338.30 \pm 13.65$ & Number of Pomc neurons \\
\hline & & Gad67 $: 391.80 \pm 4.21$ & \\
\hline & Restored: $n=8$ (5 Males, 3 Females) & Vglut2 $^{+}: 270.50 \pm 12.44$ & \\
\hline & & Gad $67^{+}: 250.10 \pm 12.95$ & \\
\hline $5 F$ (data not shown) & Control: $n=6$ (4 Males, 2 Females) & $-53.5 \pm 12.92$ & $\#$ Vglut2 ${ }^{+}, \#$ Gad67 $7^{+}$ \\
\hline Difference & Restored: $n=8$ (5 Males, 3 Females) & $20.38 \pm 8.87$ & \\
\hline \multirow[t]{3}{*}{$5 G$} & Control: $n=16$ (10 Males, 6 Females) & $1.00 \pm 0.10$ & Relative Pomc expression \\
\hline & FN $\Delta 2: n=4$ (1 Male, 3 Females) & $0.03 \pm 0.00$ & \\
\hline & Restored: $n=15$ (7 Males, 8 Females) & $1.07 \pm 0.10$ & \\
\hline \multirow[t]{5}{*}{$6 M$} & $n=6$ (3 Males, 3 Females) & Level 1: $16.73 \pm 3.10$ & $\%$ of Pomc neurons \\
\hline & & Level 2: $25.04 \pm 1.41$ & \\
\hline & & Level 3: $19.90 \pm 1.33$ & \\
\hline & & Level 4: $22.78 \pm 2.39$ & \\
\hline & & Level 5: $15.55 \pm 1.04$ & \\
\hline \multirow[t]{4}{*}{$6 N$} & $n=6$ (3 Males, 3 Females) & Pomc-only: $7.05 \pm 1.10$ & $\%$ of Pomc neurons \\
\hline & & Gad67 $7^{+}: 34.71 \pm 2.42$ & \\
\hline & & Vglut2 $^{+}: 20.62 \pm 1.31$ & \\
\hline & & Vglut2/Gad $67^{+}: 37.61 \pm 1.97$ & \\
\hline \multirow[t]{24}{*}{60} & $n=6$ (3 Males, 3 Females) & Pomc-only: & $\%$ of Pomc neurons \\
\hline & & Level 1: $2.37 \pm 0.47$ & \\
\hline & & Level 2: $1.62 \pm 0.37$ & \\
\hline & & Level 3: $1.28 \pm 0.18$ & \\
\hline & & Level 4: $1.30 \pm 0.62$ & \\
\hline & & Level 5: $0.48 \pm 0.35$ & \\
\hline & & Gad67 $7^{+}$ & \\
\hline & & Level 1: $5.44 \pm 1.80$ & \\
\hline & & Level 2: $10.26 \pm 0.52$ & \\
\hline & & Level 3: $8.80 \pm 0.71$ & \\
\hline & & Level 4: $6.47 \pm 0.76$ & \\
\hline & & Level 5: $3.75 \pm 0.70$ & \\
\hline & & Vglut2 $^{+}:$ & \\
\hline & & Level 1: $6.22 \pm 0.86$ & \\
\hline & & Level 2: $6.07 \pm 1.20$ & \\
\hline & & Level 3: $3.12 \pm 0.31$ & \\
\hline & & Level 4: $3.90 \pm 0.22$ & \\
\hline & & Level 5: $1.31 \pm 0.29$ & \\
\hline & & Vglut2/Gad67 $7^{+}:$ & \\
\hline & & Level 1: $2.69 \pm 0.54$ & \\
\hline & & Level 2: $7.09 \pm 1.00$ & \\
\hline & & Level 3: $6.71 \pm 0.91$ & \\
\hline & & Level 4: $11.12 \pm 1.58$ & \\
\hline & & Level 5: $10.02 \pm 0.44$ & \\
\hline \multirow[t]{5}{*}{$6 P$ Linear regression analysis } & $n=6$ (3 Males, 3 Females) & Pomc-only: $y=-6.795 x+40.39$ & $y=m x+b$ \\
\hline & & Gad67 $7^{+}: y=-1.977 x+25.93$ & \\
\hline & & ${\text { Gad } 67^{+}}^{+}(2-5): y=-6.449 x+43.82$ & \\
\hline & & Vglut $^{+}: \mathrm{y}=-5.784 \mathrm{x}+37.35$ & \\
\hline & & Vglut2/Gad67 $7^{+}: y=4.955 x+5.14$ & \\
\hline
\end{tabular}

Dual-label ISH for Pomc and Gad67 in restored mice identified a substantial population of $\mathrm{Gad} 67^{+}$Pomc neurons

While we did not measure any difference between control and restored mice with POMC IHC, we found significantly fewer total $\mathrm{Pomc}^{+}$cells in restored animals compared to controls using ISH (data not shown; Tables 1, 2; Fig. 5).
Dual-label ISH showed that restored mice had a greater degree of Pomc-Vglut2 and less Pomc-Gad67 overlap than was observed in control animals (Fig. 5A-E). However, as noted earlier, the total number of Pomc-labeled neurons was less in the restored animals, thus it follows that restored mice had less total Pomc-Vglut2 and Pomc-Gad67-labeled neurons than control animals (Fig. 5F). There was no difference in the cumu- 
Table 2. Statistical tests table

\begin{tabular}{lll}
\hline Figure & Data structure & Type of test \\
$1 /$ & Normal & One-way RM ANOVA \\
& distribution & (Geisser-Greenhouse correction)
\end{tabular}

Figure

$2 C$

Restored group

Figure

$3 A$

Males

Females

$3 C$

Fat mass, males

Lean mass, males

$3 D$

Fat mass, females

\section{Data structure}

Normal

distribution

Data structure

Growth curve
Two-way ANOVA

One-way ANOVA

distribution

Norma

distribution

Normal

distribution

One-way ANOVA
Type of test

Paired $t$ test

Type of test

Two-way ANOVA
Statistical data

Level: $F_{(1.65,6.60)}=1.225, p=0.34$ Animal: $F_{(4,16)}=5.038, p=8.04 \mathrm{e}-3$

Tukey's multiple comparisons test Level 1 vs level 2, $q=1.04, p=0.94$ Level 1 vs level $3, q=2.11, p=0.62$ Level 1 vs level $4, q=1.80, p=0.72$ Level 1 vs level $5, q=1.07, p=0.93$ Level 2 vs level $3, q=10.01, p=9.83 \mathrm{e}-3$ Level 2 vs level $4, q=5.23, p=8.90 \mathrm{e}-2$ Level 2 vs level $5, q=0.25, p=0.99$ Level 3 vs level $4, q=2.68, p=0.44$ Level 3 vs level $5, q=1.57, p=0.80$ Level 4 vs level $5, q=1.21, p=0.90$

Statistical data

$t=11.98, \mathrm{df}=8, p=2.17 \mathrm{e}-6$

Statistical data

Interaction $F_{(18,384)}=8.76, p<1.00 \mathrm{e}-15$ Time: $F_{(9,384)}=244, p<1.00 \mathrm{e}-15$ Genotype: $F_{(2,384)}=198.2, p<1.00 \mathrm{e}-15$ Tukey's multiple comparisons test Five weeks:

Control vs FN $\Delta 2, q=3.23, p=5.97 \mathrm{e}-2$

Control vs restored, $q=0.80, p=0.84$ FN $\Delta 2$ vs restored, $q=2.80, p=0.12$ Six weeks:

Control vs FN $\Delta 2, q=4.34, p=6.46 \mathrm{e}-3$

Control vs restored, $q=0.00, p>0.99$

FN $\Delta 2$ vs restored, $q=4.66, p=3.07 \mathrm{e}-3$ Seven weeks:

Control vs FN $\Delta 2, q=5.46, p=3.90 \mathrm{e}-4$ Control vs restored, $q=0.16, p=0.99$ FN $\Delta 2$ vs restored, $q=5.99, p=8.38 \mathrm{e}-5$

Interaction $F_{(18,294)}=19.75, p<1.00 \mathrm{e}-15$ Time: $F_{(9,294)}=158.4, p<1.00 \mathrm{e}-15$ Genotype: $F_{(2,294)}=473.2, p<1.00 \mathrm{e}-15$ Tukey's multiple comparisons test Four weeks:

Control vs FN $\Delta 2, q=1.98, p=0.34$ Control vs restored, $q=1.13, p=0.71$ FN $\Delta 2$ vs restored, $q=2.99, p=8.94 \mathrm{e}-2$ Five weeks:

Control vs FN $\Delta 2, q=4.11, p=1.10 \mathrm{e}-2$ Control vs restored, $q=1.88, p=0.38$ $\mathrm{FN} \Delta 2$ vs restored, $q=5.82, p=1.48 \mathrm{e}-4$ Six weeks:

Control vs FN $\Delta 2, q=7.80, p=2.32 \mathrm{e}-7$

Control vs restored, $q=1.69, p=0.46$

$\mathrm{FN} \Delta 2$ vs restored, $q=9.55, p=2.31 \mathrm{e}-10$

$F_{(2,23)}=138.4, p<15.1 \mathrm{e}-14$

Tukey's multiple comparisons test Control vs FN $\Delta 2, q=19.97, p=2.39 \mathrm{e}-12$

Control vs restored, $q=0.05, p>0.99$

FN $\Delta 2$ vs restored, $q=21.10, p=7.78 \mathrm{e}-13$

$F_{(2,23)}=0.167, p=0.85$

Tukey's multiple comparisons test Control vs FN $\Delta 2, q=0.50, p=0.93$

Control vs restored, $q=0.29, p=0.98$

FN $\Delta 2$ vs restored, $q=0.81, p=0.84$

$F_{(2,18)}=138.4, p=1.18 \mathrm{e}-11$

Tukey's multiple comparisons test Control vs FN $\Delta 2, q=21.01, p=4.51 \mathrm{e}-11$

Control vs restored, $q=0.33, p=0.97$

FN $\Delta 2$ vs restored, $q=21.98, p=2.09 \mathrm{e}-12$ 
Table 2. Continued

Figure
$3 D$
Lean mass, females

Gonadal fat, males

Inguinal fat, males

Gonadal fat, females

Inguinal fat, females

$4 F$

$4 F$

5 (data not shown)

\# of Pomc neurons

$5 E$

$5 E$ (data not shown)

Difference

$5 F$
Data structure

Normal

distribution

Normal

distribution

Normal

distribution

Normal

distribution

Norma

distribution

Normal

distribution

Normal

distribution

Normal

distribution

Normal

distribution

Normal

distribution

Normal

distribution

Normal

distribution
Type of test

One-way ANOVA

tatistical data

$F_{(2,18)}=6.16, p=9.17 \mathrm{e}-3$

Tukey's multiple comparisons test

Control vs FN $\Delta 2, q=3.24, p=0.08$

Control vs restored, $q=1.83, p=0.42$

FN $\Delta 2$ vs restored, $q=4.96, p=6.74 \mathrm{e}-3$

One-way ANOVA

$F_{(2,20)}=157.4, p=5.80 \mathrm{e}-14$

Tukey's multiple comparisons test

Control vs FN $\Delta 2, q=22.76, p=2.00 \mathrm{e}-12$

Control vs restored, $q=0.51, p=0.93$

FN $\Delta 2$ vs restored, $q=23.51, p=1.09 \mathrm{e}-12$

$F_{(2,19)}=354.5, p<1.00 \mathrm{e}-15$

Tukey's multiple comparisons test

Control vs FN $\Delta 2, q=34.87, p<1.00 \mathrm{e}-15$

Control vs restored, $q=0.60, p=0.91$

FN $\Delta 2$ vs restored, $q=34.40, p<1.00 \mathrm{e}-15$

One-way ANOVA

$F_{(2,18)}=173.8, p=1.70 \mathrm{e}-12$

Tukey's multiple comparisons test

Control vs FN $\Delta 2, q=24.08, p=4.26 \mathrm{e}-12$

Control vs restored, $q=0.62, p=0.90$

FN $\Delta 2$ vs restored, $q=25.06, p=2.12 \mathrm{e}-12$

One-way ANOVA

$F_{(2,17)}=57.55, p=2.70 \mathrm{e}-8$

Tukey's multiple comparisons test

Control vs FN $\Delta 2, q=13.68, p=7.26 \mathrm{e}-8$

Control vs restored, $q=0.20, p=0.99$

FN $\Delta 2$ vs restored, $q=14.49, p=3.12 \mathrm{e}-8$

Two-way ANOVA

Interaction $F_{(1,36)}=3.207, p=0.11$

Genotype: $F_{(1,36)}=3.61 \mathrm{e}-5, p=0.99$

Detection: $F_{(1,36)}=45.97, p=6.39 \mathrm{e}-8$

Tukey's multiple comparisons test

Control-A568 vs restored-A568, $q=1.57, p=0.69$

Control-A568 vs control-DAB, $q=5.24, p=3.76 \mathrm{e}-3$

Control-A568 vs restored-DAB, $q=6.65, p=2.10 \mathrm{e}-4$

Restored-A568 vs control-DAB, $q=6.92, p=1.19 \mathrm{e}-4$

Restored-A568 vs restored-DAB, $q=8.26, p=6.60 \mathrm{e}-6$

Control-DAB vs restored-DAB, $q=1.74, p=0.61$

One-way ANOVA

$F_{(2,51)}=24.9, p=2.85 \mathrm{e}-8$

Tukey's multiple comparisons test

A568 vs DAB, $q=9.97, p=1.35 \mathrm{e}-8$

A548 vs A488, $q=4.58, p=5.87 \mathrm{e}-3$

$\mathrm{DAB}$ vs $\mathrm{A} 488, q=4.74, p=4.31 \mathrm{e}-3$

Student's $t$ test

$t=10.75, \mathrm{df}=13, p=7.76 \mathrm{e}-8$

Two-way RM ANOVA

Interaction $F_{(1,12)}=20.23, p=7.30 \mathrm{e}-4$

Genotype: $F_{(1,12)}=3.181, p=9.98 \mathrm{e}-2$

Marker: $F_{(1,12)}=1.363, p=0.27$

Sidak's multiple comparisons test

Control-Vglut2 ${ }^{+}$vs restored-Vglut $2^{+}, t=4.37, p=4.10 \mathrm{e}-4$

Control-Gad67 ${ }^{+}$vs restored-Gad67 $7^{+}, t=1.76, p=0.17$

Control-Vglut2 ${ }^{+}$vs control-Gad67 ${ }^{+}, t=3.75, p=5.56 \mathrm{e}-3$

Restored-Vglut2 ${ }^{+}$vs restored-Gad $67^{+}, t=2.54, p=5.09 \mathrm{e}-2$

Student's $t$ test

$t=4.498, \mathrm{df}=12, p=7.30 \mathrm{e}-4$

Two-way RM ANOVA

Interaction $F_{(1,12)}=23.86, p=3.76 \mathrm{e}-4$

Genotype: $F_{(1,12)}=46.4, p=1.87 \mathrm{e}-5$

Marker: $F_{(1,12)}=4.797, p=4.90 \mathrm{e}-2$

Sidak's multiple comparisons test

Control-Vglut2 ${ }^{+}$vs restored-Vglut2 ${ }^{+}, t=3.96, p=1.17 \mathrm{e}-3$

Control-Gad $67^{+}$vs restored-Gad67 $7^{+}, t=8.27, p=3.50 \mathrm{e}-8$

Control-Vglut2 ${ }^{+}$vs control-Gad67 ${ }^{+}, t=4.68, p=1.07 \mathrm{e}-3$

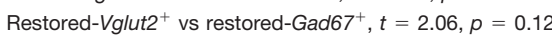

$t=4.884, \mathrm{df}=12, p=3.76 \mathrm{e}-4$

(Continued) 
Table 2. Continued

\begin{tabular}{lll}
\hline $\begin{array}{l}\text { Figure } \\
5 G\end{array}$ & $\begin{array}{l}\text { Data structure } \\
\text { Normal } \\
\text { distribution }\end{array}$ & $\begin{array}{l}\text { Type of test } \\
\text { One-way ANOVA }\end{array}$ \\
$6 M$ & $\begin{array}{l}\text { Normal } \\
\text { distribution }\end{array}$ & Two-way RM ANOVA
\end{tabular}

\section{distribution}

Norma

distribution
Statistical data

$F_{(2,32)}=10.63, p=2.88 \mathrm{e}-4$

Tukey's multiple comparisons test

Control vs FN $\Delta 2, q=5.96, p=5.43 \mathrm{e}-4$

Control vs restored, $q=0.65, p=0.89$

FN $\Delta 2$ vs restored, $q=6.34, p=2.54 \mathrm{e}-4$

Interaction $F_{(12,60)}=13.10, p=1.19 \mathrm{e}-12$

Neurotransmitter: $F_{(3,15)}=47.14, p=7.16 \mathrm{e}-8$

Level: $F_{(4,20)}=3.16, p=3.63 \mathrm{e}-2$

Tukey's multiple comparisons test

Level 1 vs level 2, $q=3.71, p=0.10$

Level 1 vs level $3, q=1.42, p=0.85$

Level 1 vs level $4, q=2.70, p=0.35$

Level 1 vs level $5, q=0.52, p>0.99$

Level 2 vs level $3, q=2.29, p=0.50$

Level 2 vs level $4, q=1.01, p=0.95$

Level 2 vs level $5, q=4.23, p=5.02 \mathrm{e}-2$

Level 3 vs level $4, q=1.28, p=0.89$

Level 3 vs level $5, q=1.94, p=0.65$

Level 4 vs level $5, q=3.22, p=0.19$

Interaction $F_{(12,60)}=13.10, p=1.19 \mathrm{e}-12$

Neurotransmitter: $F_{(3,15)}=47.14, p=7.16 \mathrm{e}-8$

Level: $F_{(4,20)}=3.16, p=3.63 \mathrm{e}-2$

Tukey's multiple comparisons test

Vglut2 $^{+}$vs Gad67 ${ }^{+}, q=6.87, p=1.07 \mathrm{e}-3$

Vglut2 $^{+}$vs Vglut2/Gad67 ${ }^{+}, q=8.29, p=1.66 \mathrm{e}-4$

Vglut2 $^{+}$vs Pomc-only, $q=6.62, p=1.51 \mathrm{e}-3$

${\text { Gad } 67^{+} \text {vs Vglut2/Gad67 }}^{+}, q=1.42, p=0.75$

Gad67 $7^{+}$vs Pomc-only, $q=13.49, p=5.09 \mathrm{e}-7$

Vglut2/Gad $67^{+}$vs Pomc-only, $q=14.90, p=1.37 \mathrm{e}-7$

Interaction $F_{(12,60)}=13.10, p=1.19 \mathrm{e}-12$

Neurotransmitter: $F_{(3,15)}=47.14, p=7.16 \mathrm{e}-8$

Level: $F_{(4,20)}=3.16, p=3.63 \mathrm{e}-2$

Tukey's multiple comparisons test Vglut2 $^{+}$:

Level 1 vs level 2, $q=0.23, p=0.99$

Level 1 vs level $3, q=4.54, p=1.75 \mathrm{e}-2$

Level 1 vs level $4, q=3.39, p=0.13$

Level 1 vs level $5, q=7.18, p=3.80 \mathrm{e}-5$

Level 2 vs level $3, q=4.31, p=2.71 \mathrm{e}-2$

Level 2 vs level $4, q=3.16, p=0.18$

Level 2 vs level $5, q=6.96, p=6.80 \mathrm{e}-5$

Level 3 vs level $4, q=1.15, p=0.93$

Level 3 vs level $5, q=2.64, p=0.35$

Level 4 vs level $5, q=3.79, p=6.85 \mathrm{e}-2$

Gad67+:

Level 1 vs level 2, $q=7.05, p=5.33 \mathrm{e}-5$

Level 1 vs level 3, $q=4.90, p=8.36 \mathrm{e}-3$

Level 1 vs level $4, q=1.50, p=0.83$

Level 1 vs level $5, q=7.18, p=0.41$

Level 2 vs level $3, q=2.15, p=0.55$

Level 2 vs level $4, q=5.55, p=2.03 \mathrm{e}-3$

Level 2 vs level $5, q=9.53, p=6.85 \mathrm{e}-8$

Level 3 vs level $4, q=3.40, p=0.13$

Level 3 vs level $5, q=7.38, p=2.26 \mathrm{e}-5$

Level 4 vs level $5, q=3.98, p=4.98 \mathrm{e}-2$

Pomc-only:

Level 1 vs level 2, $q=1.10, p=0.94$

Level 1 vs level $3, q=1.59, p=0.79$

Level 1 vs level $4, q=1.56, p=0.80$

Level 1 vs level $5, q=2.77, p=0.30$

Level 2 vs level $3, q=0.49, p=0.99$

Level 2 vs level $4, q=0.46, p=0.99$

Level 2 vs level $5, q=1.67, p=0.76$

Level 3 vs level $4, q=0.03, p=0.99$

Level 3 vs level $5, q=1.18, p=0.92$

Level 4 vs level $5, q=1.21, p=0.91$ 
Table 2. Continued

\section{Figure}

Data structure
Type of test

Two-way RM ANOVA
Normal

distribution
Statistical data

Vglut2/Gad67 ${ }^{+}$

Level 1 vs level $2, q=6.44, p=2.51 \mathrm{e}-4$

Level 1 vs level $3, q=5.88, p=9.63 \mathrm{e}-4$

Level 1 vs level $4, q=12.31, p=5.13 \mathrm{e}-11$

Level 1 vs level $5, q=10.71, p=2.63 \mathrm{e}-9$

Level 2 vs level $3, q=0.56, p=0.99$

Level 2 vs level $4, q=5.87, p=9.77 \mathrm{e}-4$

Level 2 vs level $5, q=4.27, p=2.92 \mathrm{e}-2$

Level 3 vs level $4, q=6.43, p=5.54 \mathrm{e}-4$

Level 3 vs level $5, q=4.83, p=9.68 \mathrm{e}-3$

Level 4 vs level $5, q=1.60, p=0.79$

Level 1:

Vglut2 $^{+}$vs Gad67 ${ }^{+}, q=1.14, p=0.85$

Vglut2 $^{+}$vs Vglut2/Gad67 ${ }^{+}, q=5.16, p=3.00 \mathrm{e}-3$

Vglut $^{+}$vs Pomc-only, $q=5.63, p=1.05 \mathrm{e}-3$

${\text { Gad } 67^{+}}^{+}$vs Vglut2/Gad67 ${ }^{+}, q=4.02, p=3.02 \mathrm{e}-2$

Gad67 ${ }^{+}$vs Pomc-only, $q=4.49, p=1.23 \mathrm{e}-2$

Vglut2/Gad67 ${ }^{+}$vs Pomc-only, $q=0.47, p=0.99$

Level 2:

Vglut2 $^{+}$vs Gad67 ${ }^{+}, q=6.14, p=3.18 \mathrm{e}-4$

Vglut2 $^{+}$vs Vglut2/Gad67 $7^{+}, q=1.50, p=0.72$

Vglut2 $^{+}$vs Pomc-only, $q=6.51, p=1.29 \mathrm{e}-4$

${\text { Gad } 67^{+} \text {vs Vglut2/Gad67 }}^{+}, q=4.64, p=9.16 \mathrm{e}-3$

Gad67 ${ }^{+}$vs Pomc-only, $q=12.64, p=2.74 \mathrm{e}-11$

Vglut2/Gad $67^{+}$vs Pomc-only, $q=8.00, p=2.67 \mathrm{e}-6$

Level 3:

Vglut2 $^{+}$vs Gad67 $7^{+}, q=8.30, p=1.20 \mathrm{e}-6$

Vglut2 $^{+}$vs Vglut2/Gad67 ${ }^{+}, q=5.25, p=2.48 \mathrm{e}-3$

Vglut2 $^{+}$vs Pomc-only, $q=2.68, p=0.24$

Gad $67^{+}$vs Vglut2/Gad67 ${ }^{+}, q=3.05, p=0.15$

Gad $67^{+}$vs Pomc-only, $q=10.99, p=7.50 \mathrm{e}-10$

Vglut2/Gad $67^{+}$vs Pomc-only, $q=7.94, p=3.19 \mathrm{e}-6$

Level 4:

Vglut2 $^{+}$vs Gad67 ${ }^{+}, q=3.75, p=4.92 \mathrm{e}-2$

Vglut2 $^{+}$vs Vglut2/Gad67 ${ }^{+}, q=10.53, p=2.60 \mathrm{e}-9$

Vglut2 $^{+}$vs Pomc-only, $q=3.80, p=4.45 \mathrm{e}-2$

${\text { Gad } 67^{+}}^{+}$vs Vglut2/Gad67 ${ }^{+}, q=6.78, p=6.41 \mathrm{e}-5$

Gad $67^{+}$vs Pomc-only, $q=7.55, p=8.88 \mathrm{e}-6$

Vglut2/Gad $67^{+}$vs Pomc-only, $q=14.33, p=2.00 \mathrm{e}-11$

Level 5:

Vglut2 $^{+}$vs Gad67 ${ }^{+}, q=3.56, p=6.73 \mathrm{e}-2$

Vglut2 $^{+}$vs Vglut2/Gad67 $7^{+}, q=12.73, p=2.58 \mathrm{e}-11$

Vglut2 $^{+}$vs Pomc-only, $q=1.22, p=0.83$

Gad67 $^{+}$vs Vglut2/Gad67 ${ }^{+}, q=9.17, p=1.13 \mathrm{e}-7$

Gad67 ${ }^{+}$vs Pomc-only, $q=4.78, p=6.89 \mathrm{e}-3$

Vglut2/Gad6 $7^{+}$vs Pomc-only, $q=13.94, p=2.01 \mathrm{e}-11$

Interaction $F_{(12,60)}=11.47, p=1.69 \mathrm{e}-11$

Neurotransmitter: $F_{(3,15)}=2.105, p=0.14$

Level: $F_{(4,20)}=3.205, p=3.46 \mathrm{e}-2$

Tukey's multiple comparisons test

Vglut2 ${ }^{+}$:

Level 1 vs level 2, $q=0.45, p=0.99$

Level 1 vs level $3, q=5.48, p=2.42 \mathrm{e}-3$

Level 1 vs level $4, q=3.95, p=5.22 \mathrm{e}-2$

Level 1 vs level $5, q=8.61, p=8.65 \mathrm{e}-7$

Level 2 vs level $3, q=5.02, p=6.49 \mathrm{e}-3$

Level 2 vs level $4, q=3.50, p=0.11$

Level 2 vs level $5, q=8.16, p=2.92 \mathrm{e}-6$

Level 3 vs level $4, q=1.52, p=0.82$

Level 3 vs level $5, q=3.13, p=0.19$

Level 4 vs level $5, q=4.65, p=1.39 \mathrm{e}-2$

Gad67 ${ }^{+}$:

Level 1 vs level 2, $q=5.36, p=3.13 \mathrm{e}-3$

Level 1 vs level $3, q=3.76, p=7.27 \mathrm{e}-2$

Level 1 vs level $4, q=1.37, p=0.87$

Level 1 vs level $5, q=1.54, p=0.81$

Level 2 vs level $3, q=1.60, p=0.79$

Level 2 vs level $4, q=4.00, p=4.85 \mathrm{e}-2$

Level 2 vs level $5, q=6.90, p=7.79 \mathrm{e}-5$ 
Table 2. Continued

Figure

Data structure
Type of test

Linear regression

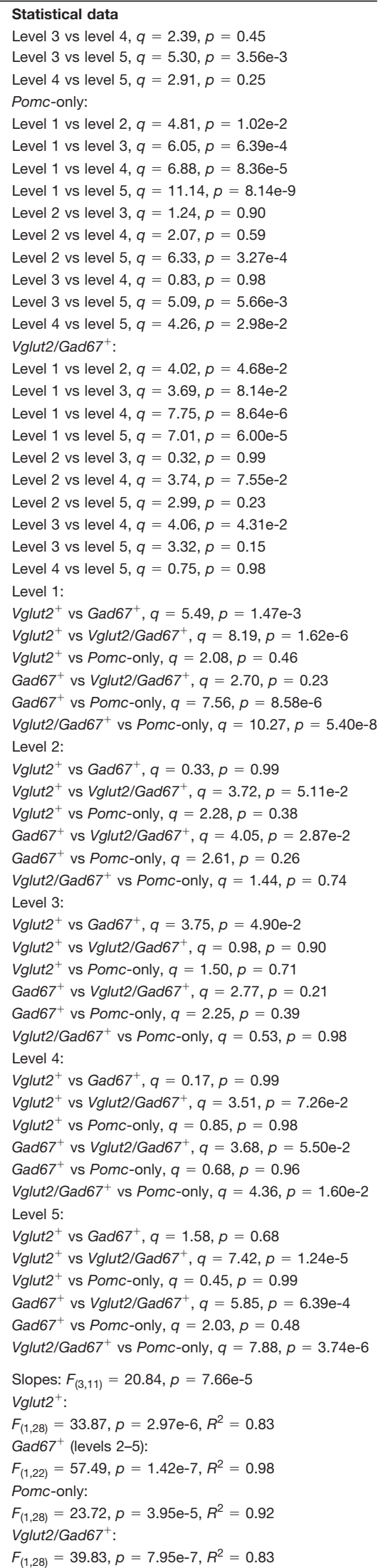



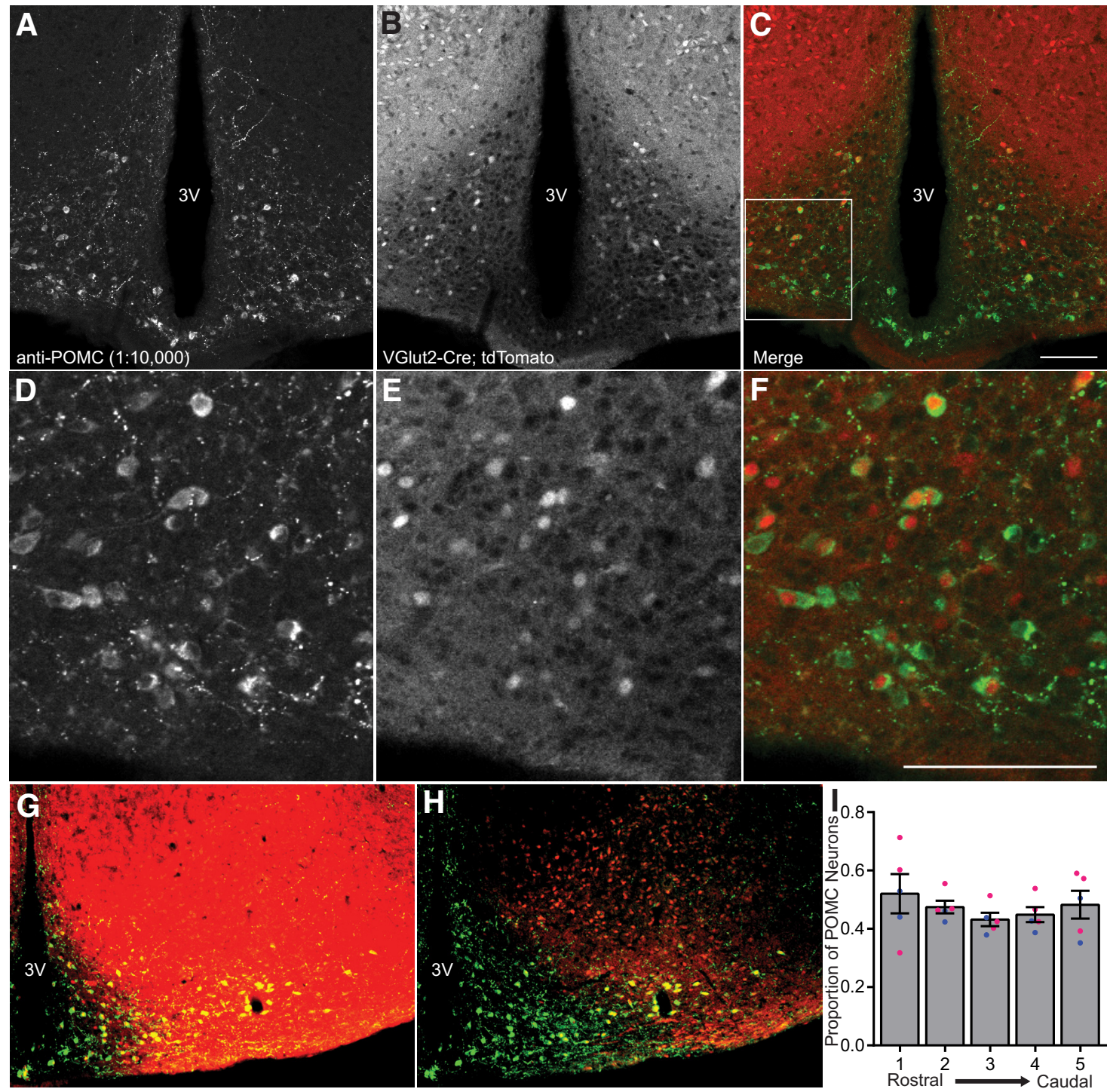

Figure 1. Genetic lineage trace of Vglut2 expression and overlap with POMC IHC. $\boldsymbol{A}-\boldsymbol{C}$, Confocal images of POMC immunoreactivity (green) from a male mouse $(\boldsymbol{A})$, Vglut2-Cre-mediated tdTomato expression (red) $(\boldsymbol{B})$, and overlap between the two signals $(\boldsymbol{C})$. $\boldsymbol{D}-\boldsymbol{F}$, $40 \times$ zoom of the inset outlined in panel $\boldsymbol{C}$ with the same signals as in $\boldsymbol{A}-\boldsymbol{C}$. $\mathbf{G}-\boldsymbol{H}, \mathbf{2 0} \times$ epifluorescent image from a female mouse with post hoc processing to identify tdTomato ${ }^{+}$-POMC overlap (yellow) in the medial $(\boldsymbol{G})$ and lateral $(\boldsymbol{H})$ ARC. $\boldsymbol{I}$, Quantification of the proportion of tdTomato ${ }^{+}-\mathrm{POMC}$ overlap throughout the rostral-caudal ARC axis. Male data for each group represented by filled blue circles and female data shown by filled pink circles. The scale bars represent $100 \mu \mathrm{m}$. 3V, third ventricle.

lative sum of the percentage of Pomc-Vglut2 and Pomc-Gad67 cells between restored and control animals, indicating that each group possesses a proportionately comparable population of dual Vglut2 ${ }^{+} / \mathrm{Gad} 67^{+}$phenotype Pomc neurons. qRTPCR for relative Pomc expression was performed on CDNA derived from RNA extracted from the medial-basal hypothalamus. Despite detecting fewer Pomc neurons with ISH, there was no difference in total steady-state Pomc mRNA levels between restored and control animals, and levels in both groups were $\sim 25$-fold greater than FN $\Delta 2$ mice (Fig. $5 G$ ).

Triple-label ISH in WT mice showed distinct Pomc-only, Gad $67^{+} /$Pomc, Vglut2 ${ }^{+} /$Pomc, and Gad $67^{+} /$Vglut2 $^{+} /$ Pome neuron subpopulations with varying anatomic distribution along the rostral-caudal ARC axis

The individual hybridization signals for Pomc, Gad67, and Vglut2 from a representative hemi-section of the medial basal hypothalamus including the $\mathrm{ARC}$ and $\mathrm{VMH}$ are shown in Figure $6 A-C$. The merged image is shown in Figure $6 D$. Both Pomc and Gad67 expression are primarily located in the ARC while Vglut2 mRNA is most densely located in the VMH. Neurons of interest were identified as shown in representative higher magnification sections from the rostral Arc (Fig. $6 E-H$ ) and the caudal ARC (Fig. $6 /-L$ ). There was a main effect on Pomc neuron counts along the rostral-caudal axis of the ARC. However, none of the Levels differed significantly from one another after post hoc multiple comparisons (Fig. 6M). Of the possible phenotypic combinations of Pomc expression overlapping with the two neurotransmitter markers throughout the entirety of the ARC, the Pomc-only cells constituted the smallest group, followed by double Pomc/Vglut2 ${ }^{+}$and 
A

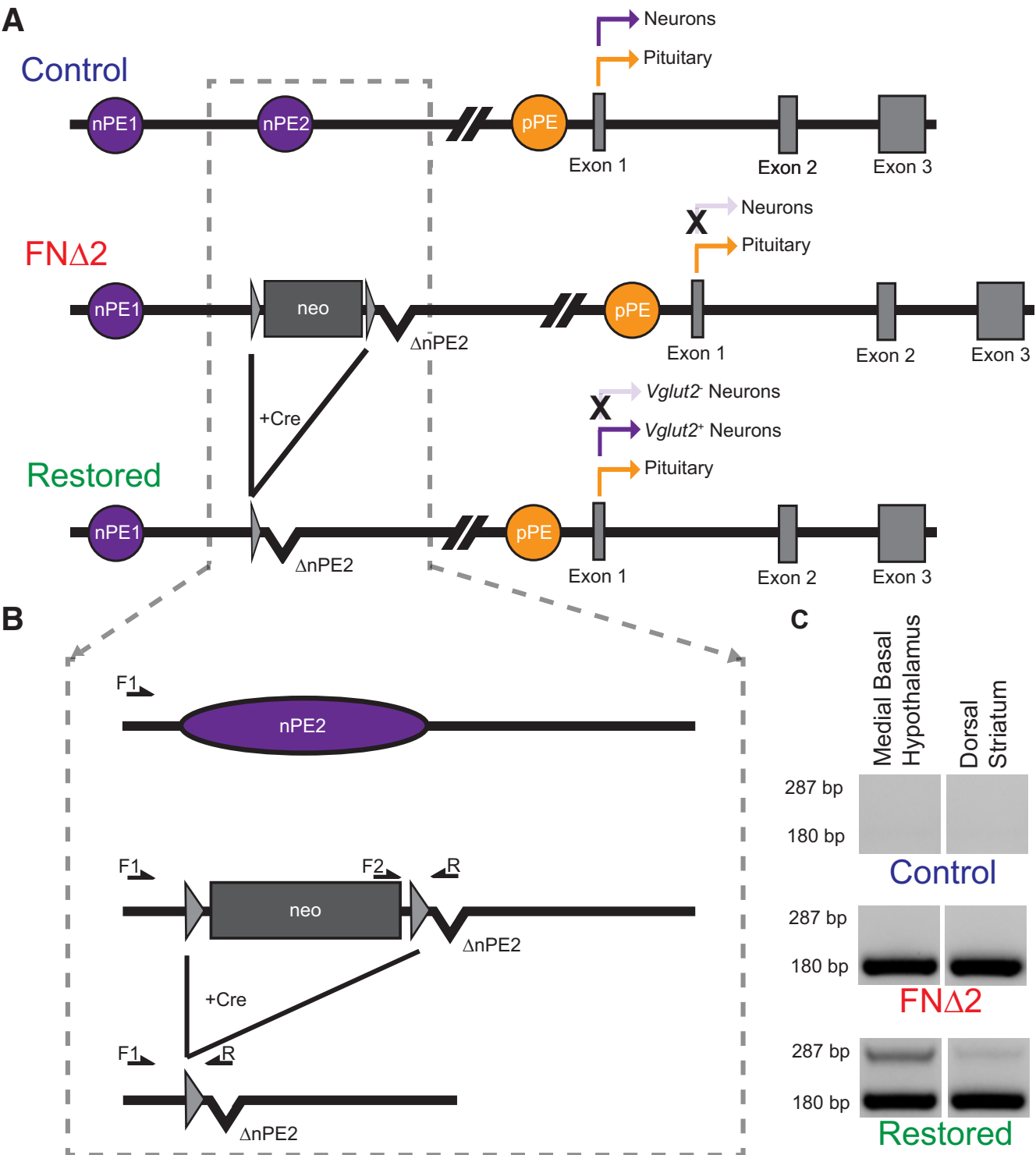

Figure 2. Vglut2-ires-Cre-specific recombination of the Pomc FN $\Delta 2$ allele. $\boldsymbol{A}$, Schematic representing the WT Pomc locus (control), the presence of the Cre-excisable floxed neomycin cassette along with the knock-out of nPE2 (FN $\Delta 2$ ), and the FN $\Delta 2$ allele after Cre-mediated excision leaving only the knock-out of nPE2 (restored). $\boldsymbol{B}$, Hybridization location of the three oligonucleotide primer set used to assess the integrity of the Cre-mediated recombination. The forward 1 (F1) primer hybridizes upstream of nPE2, the forward 2 (F2) primer hybridizes to the intact neomycin cassette, and the reverse (R) primer is specific to the knocked out nPE2 locus. $\boldsymbol{C}$, Verification of Cre-mediated genetic excision of the floxed-neomycin cassette from the Pomc neural enhancer locus in restored mice. The intensity of the recombined band (PCR product of F1 and R; $287 \mathrm{bp}$ ) versus the non-recombined band (PCR product of F2 and $\mathrm{R} ; 180 \mathrm{bp})$ was much stronger in the medial basal hypothalamus than in the dorsal striatum. $(P<0.00001$, see Table 2$)$.

double Pomc/Gad67 $7^{+}$neurons. Triple Pomc/Vglut2 ${ }^{+} /$ Gad $67^{+}$cells comprised the largest group. Except for the Pomc/Gad67 $7^{+}$count compared to the Pomc/Vglut2/ Gad6 $67^{+}$population, the size of every group was significantly different from every other group (Fig. $6 \mathrm{~N}$ ). Analysis of linear regression revealed several patterns that emerged along the rostral-caudal axis of the ARC. The Pomc-only and Vglut2 ${ }^{+}$populations exhibited a similar trend of having the highest presence at the rostral end of the ARC and the lowest at the caudal end, while the Vglut2/Gad $67^{+}$cells showed the opposite pattern. The Gad $67^{+}$population did not exhibit a linear trend like the other groups, due to a sharp increase in expression between the two most rostral ARC positions. However, analyzing levels 2 through 5 showed that their numbers also diminished toward the caudal ARC (Fig. 6O,P).

We also observed a small number of dual Vglut2/Gad $67^{+}$ cells in the ARC that were not positive for Pomc mRNA. The phenotypic identity of these cells is not known. Furthermore, the density of non-Pomc dual Vglut2/Gad67 $7^{+}$cells was higher in the DMN (data not shown). Together, these data indicate that neuron populations in the hypothalamus, other than POMC neurons, may also exhibit both glutamatergic and GABAergic characteristics. 

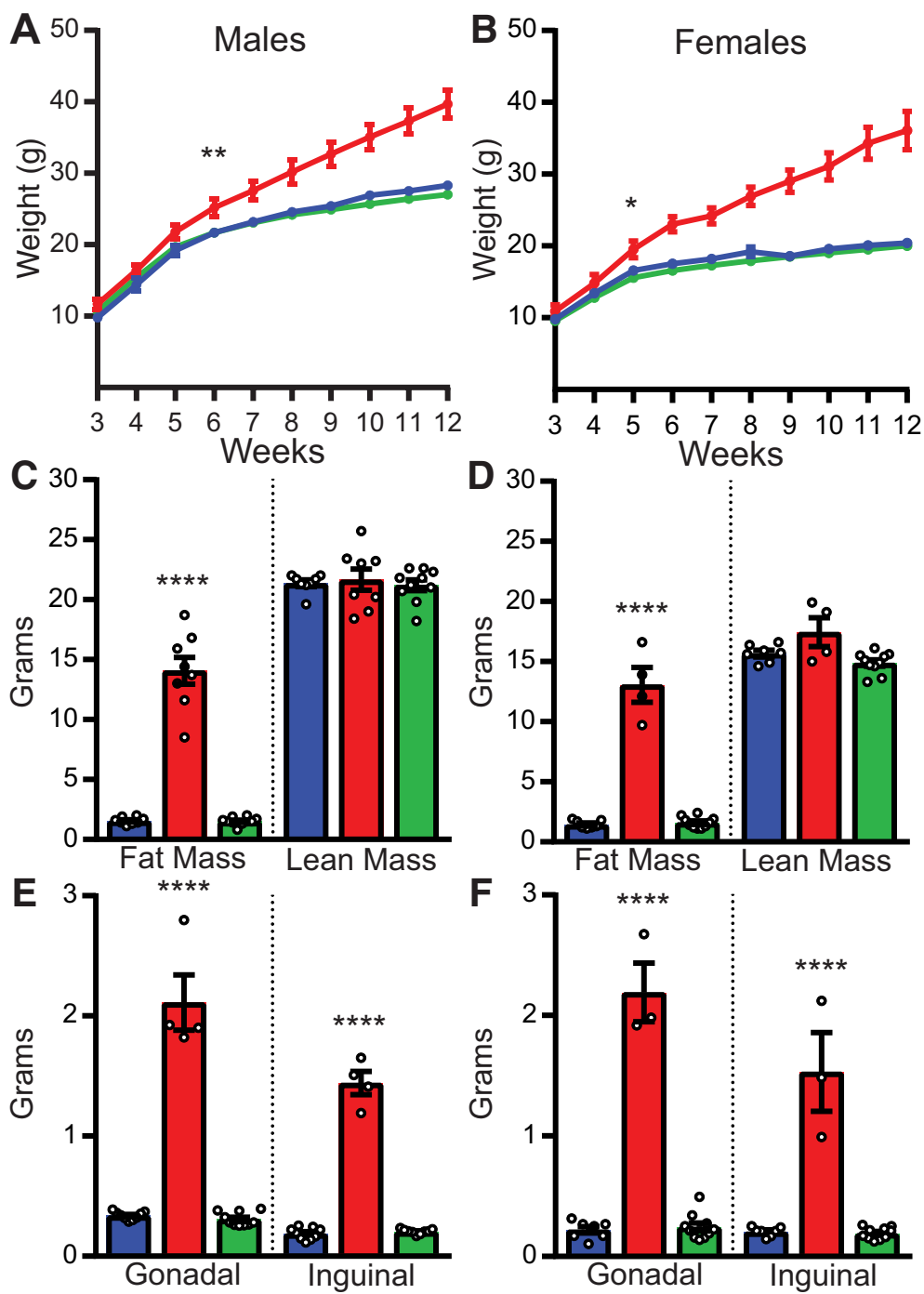

Figure 3. Vglut2-ires-Cre-mediated recombination of Pomc normalizes body composition. $\boldsymbol{A}$, Growth curves from male mice. FN $\Delta 2$ (red line) mice significantly diverged from both control (blue line) and restored (green line) mice by six weeks of age. $\boldsymbol{B}$, Growth curves from female mice. FN $\Delta 2$ mice significantly diverged from both control and restored mice by five weeks of age. $\boldsymbol{C}, \boldsymbol{D}$, NMR assessment of body composition. Male (C) and female (D) FN $\Delta 2$ mice (red bars) had substantially more body fat than control (blue bars) or restored (green bars) mice, while there were no differences in lean mass. $\boldsymbol{E}, \boldsymbol{F}$, Weight of gonadal and inguinal fat depots. Male (E) and female (F) FN $\Delta 2$ mice (red bars) had substantially larger fat depots (both gonadal and inguinal) than control (blue bars) or restored (green bars) mice. $* p<0.05, * * p<0.01, * * * * p<0.001$

\section{Discussion}

The global impact of POMC peptides on metabolic homeostasis, body composition, and feeding behavior is widely recognized. However, studies investigating the fast synaptic transmission roles that POMC neurons serve are in their early stages. The initial rationale for conducting the current study was to decipher the physiological significance of glutamate-producing POMC neurons, using an experimental paradigm previously used to study leptin receptor POMC neurons (Lam et al., 2015a) and 5HT-2cR POMC neurons (Burke et al., 2016), by selective induction of Pomc transcription in these cell populations during hypothalamic development. We sought to characterize the function of a specific neuronal subset, but what we uncovered instead is a broader phenomenon of neural adaptation or plasticity.
The complete prevention of the obesity phenotype of FN $\Delta 2$ mice by Vglut2-ires-Cre induction of Pomc expression in hypothalamic neurons did not support our initial hypothesis that a subpopulation of glutamatergic POMC neurons would have selective effects on energy homeostasis. It is possible that more extensive metabolic phenotyping of the restored mice, including environmental challenges such as a high-fat diet, might have unveiled subtle alterations from control mice. However, the existing data for body weight growth over 12 weeks, normal body composition and normalized steady state Pomc mRNA levels at age 12 weeks, combined with the observed developmental alterations in neurotransmitter markers, suggested that additional metabolic phenotyping was unlikely to be informative or fully interpretable. Previous work indicated that Pomc mRNA levels above a threshold of $30 \%$ 

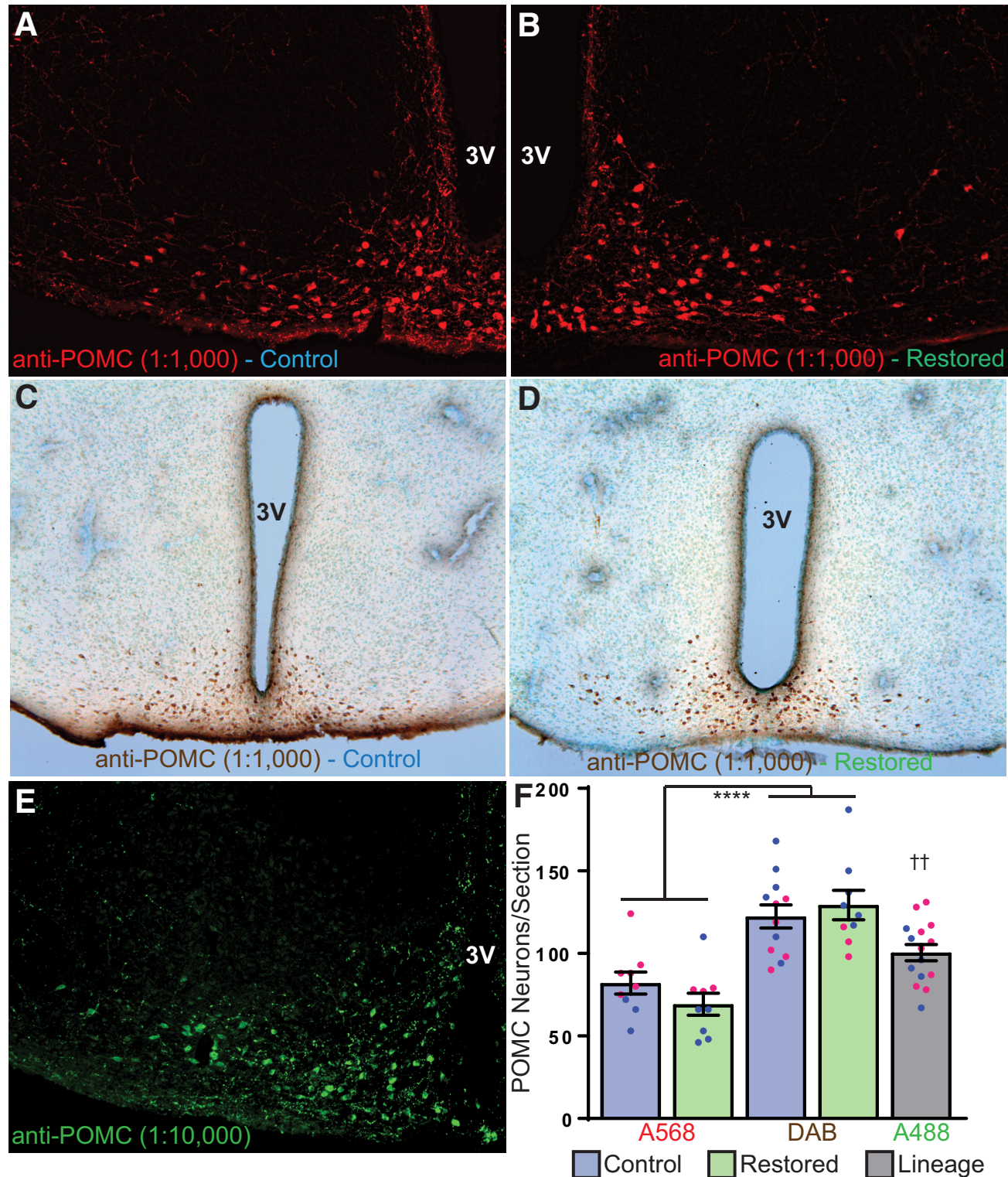

Figure 4. IHC for POMC cell counts in control and restored mice, and from VGlut2-Cre; tdTomato animals. A, POMC-IR in a male control mouse detected with an Alexa Fluor 568 (red) secondary antibody (1:500). B, POMC-IR in a male restored mouse detected with an Alexa Fluor 568 (red) secondary antibody (1:500). C, POMC-IR in a female control mouse detected with biotinylated secondary antibody (1:500) and visualized with a diaminobenzidine (DAB) reaction (brown). $\boldsymbol{D}$, POMC-IR in a female restored mouse detected with biotinylated secondary antibody $(1: 500)$ and visualized with a DAB reaction (brown). $\boldsymbol{E}$, POMC-IR in a female Vglut2-Cre; tdTomato mouse detected with an Alexa Fluor 488 (green) secondary antibody (1:500; mirrored section from Fig. 1G,H). F, POMC neuron cell counts from sections (three per mouse). There was no difference between control (blue bars) or restored (green bars) mice, but only in the method of secondary labeling used. Male data for each group represented by filled blue circles and female data shown by filled pink circles; $* * * * p<1 \mathrm{e}-7$, denotes the difference between all A568 counts compared to all DAB counts, $\dagger+p<0.01$, denotes the difference between all A488 counts compared to all A568 and DAB cell counts. 3V, third ventricle.

of control levels and evenly distributed spatially across the rostral-caudal axis of the ARC produced only a mild obesity phenotype in low fat chow fed mice (Lam et al., 2015b). Furthermore, Pomc mRNA levels above $50 \%$ of control levels protected mice from obesity, even when challenged with a high-fat diet (Lam et al., 2015b).

A first line of evidence for fast neurotransmitter plasticity within the POMC neuronal network arises from a discrepancy that we found between Pomc restoration in FN $\Delta 2$ mice and the Vglut2 lineage trace experiments, even though each study was dependent on the same Cre-driver mouse strain. On one hand, we found a nearly complete restoration in the number of POMCexpressing neurons, indicating that all of those neurons either contemporaneously expressed or were derived from cells that had expressed Vglut2 at some timepoint. On the other hand, we found that only half of POMC immunoreactive cells were also positive for the lineage trace, suggesting that all POMC neurons could not have expressed or be derived from cells that were 

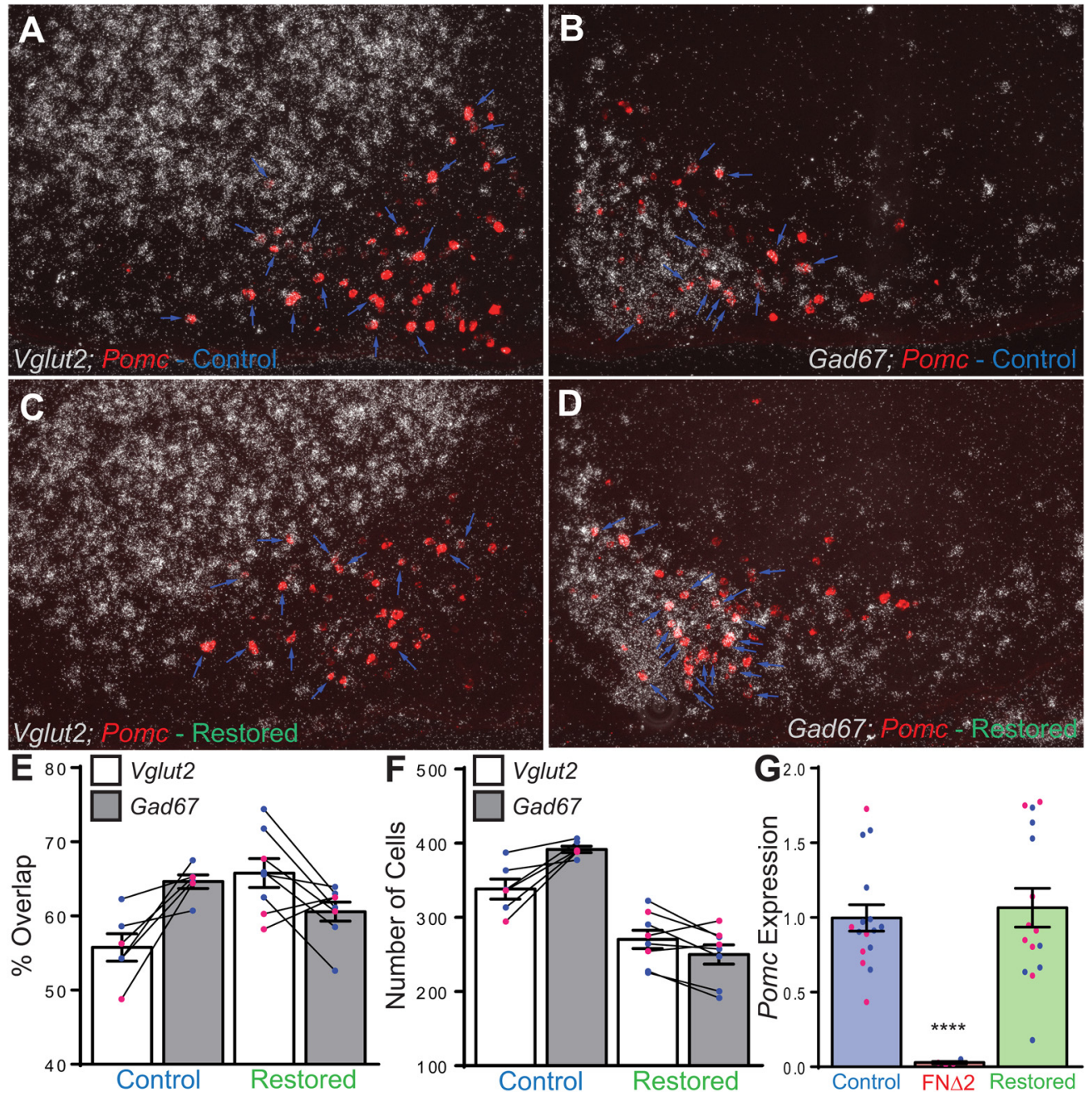

Figure 5. Dual-label ISH for Pomc and Vglut2 or Gad67, and relative Pomc expression in the medial basal hypothalamus of control and restored mice. A, ISH for Vglut2 (silver grains) and Pomc (red) in a female control mouse. Note that the green fluorescence of Alexa Fluor 488 used to detect Pomc was pseudocolored to red for these images. B, ISH for Gad67 (silver grains) and Pomc (red) in a female control mouse. C, ISH for Vglut2 (silver grains) and Pomc (red) in a female restored mouse. D, ISH for Gad67 (silver grains) and Pomc (red) in a female restored mouse. In panels $\boldsymbol{A}-\boldsymbol{D}$, blue arrows indicate overlap between Pomc and the silver grain (Vglut2 or Gad67) signal. E, Degree of overlap between Pomc and Vglut2 (white bars) or Gad67 (grey bars) in control and restored mice, each animal's Vlgut2/Pomc and Gad67/Pomc overlap percentage is connected by the solid black lines. F, Cell count of overlap between Pomc and Vglut2 (white bars) or Gad67 (grey bars) in control and restored mice, each animal's Vlgut2/Pomc and Gad67/Pomc overlap count is connected by the solid black lines. G, Relative qRT-PCR of Pomc expression in the medial-basal hypothalamus of control (blue bar, left), $\mathrm{FN} \Delta 2$ (red bar, middle), and restored (green bar, right) mice. Male data are represented by filled blue circles and female data by filled pink circles.

Vglut2 $^{+}$at some point. We can only speculate about the exact mechanism underlying these differences, but there may be a combination of multiple processes including sensitivity issues in the methods of detection between the two studies. Furthermore, distinct floxed alleles may have different thresholds for recombination by the same Cre-driver strain (Song and Palmiter, 2018).

It is possible that the restored mice had differences in developmental timing en route to normalization of the POMC system, which were not evident in measurements of body mass and composition. In the Vglut2 lineage trace, normal POMC function should be intact and allow POMC neurons to follow a normal developmental program, result- ing in an even split between tdTomato ${ }^{+}$and tdTomato POMC neurons. In contrast, the Pomc-restoration experiment used FN $\Delta 2$ mice that are incapable of transcribing Pomc in neurons, and arguably undergo an altered developmental program that differentially impacts the hypothalamic landscape and ultimately global metabolic function. This fact is evident in the phenotypic traits of the latter mice: morbid obesity, hyperphagia, hypolocomotion, insulin resistance and alterations in glucose tolerance and glycosuria (Bumaschny et al., 2012; Chhabra et al., 2016a,b). However, when Pomc expression was induced in these mice by the Vglut2-IRES-Cre driver, the animals were phenotypically normal and had the same proportion of dual-labeled PomcGad $67^{+}$cells and POMC immunoreactive cells as control 

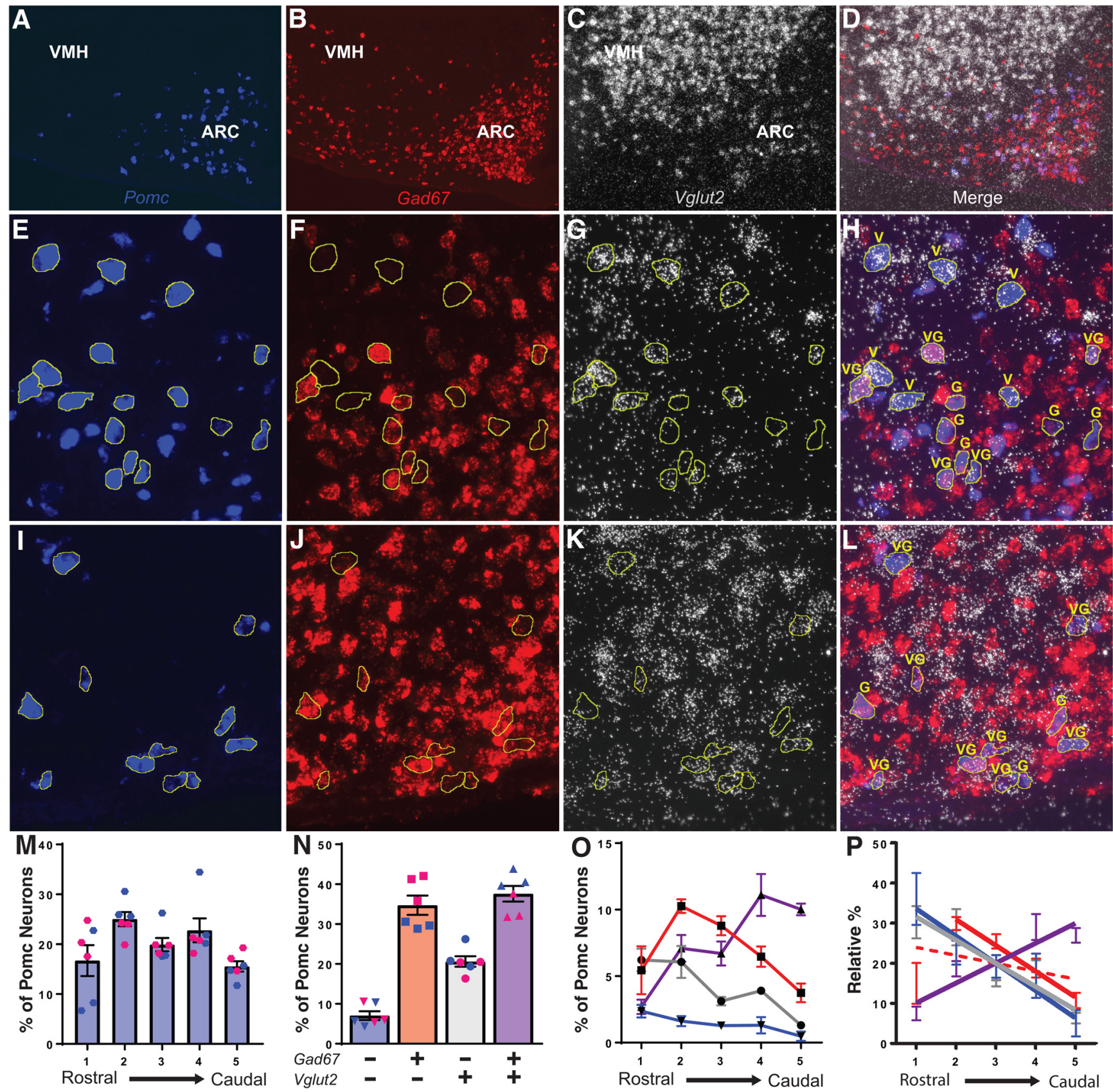

Figure 6. Triple-label ISH for Pomc $(\boldsymbol{A}, \boldsymbol{E}, \boldsymbol{I})$, Gad67 (B, $\boldsymbol{F}, \boldsymbol{J})$, Vglut2 (C,G,K), and overlaid signals $(\boldsymbol{D}, \boldsymbol{H}, \boldsymbol{L})$ in WT mice throughout the rostral-caudal ARC axis. $\boldsymbol{A}-\boldsymbol{D}$, Low-magnification image of ISH signal for Pomc, Gad67, Vglut2, and the overlay of all signals from a male mouse. Note that the green fluorescence of Alexa Fluor 488 used to detect Pomc was pseudocolored to blue for these images. $\boldsymbol{E}-\boldsymbol{H}, 40 \times$ images from the rostral ARC from a male mouse with Pomc neuron profiles outlined in yellow. $\boldsymbol{I}-\boldsymbol{L}, 40 \times$ images from the

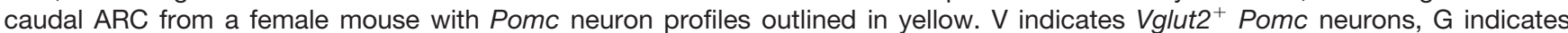
Gad67 ${ }^{+}$Pomc neurons, and VG indicates Vglut2/Gad67 ${ }^{+}$Pomc neurons. $\boldsymbol{M}$, The distribution of Pomc neurons along the rostralcaudal ARC axis. N, The overall percentages of Pomc-only (blue bar with filled inverted triangles), Gad67 $7^{+}$(red bar with filled squares), Vglut2 $^{+}$(grey bar with filled circles), and Vglut2/Gad67 ${ }^{+}$(purple bar with filled triangles) Pomc neurons in the arcuate nucleus. Male data are represented by filled blue symbols and female data by filled pink symbols. $\boldsymbol{O}$, The percentages of Pomc-only (blue line with filled inverted triangles), Gad67 ${ }^{+}$(red line with filled squares), Vglut2 ${ }^{+}$(grey line with filled circles), and Vglut2/Gad67 ${ }^{+}$(purple line with filled triangles) Pomc neurons at each coronal level 1 to 5 along the rostral-caudal ARC axis. $\boldsymbol{P}$, Linear regression analysis of the relative percentage of each phenotypic category of Pomc neurons along the rostral-caudal ARC axis [Pomc-only solid blue line, $\mathrm{Gad}_{67^{+}}$dotted red line, Gad67 ${ }^{+}$(levels 2-5) solid red line, Vglut2 ${ }^{+}$solid grey line, Vglut2/Gad67 ${ }^{+}$solid purple line].

animals. Both of these results were unanticipated, we expected to restore function to only a subset of POMC neurons and that relatively few of them would be Gad67 $7^{+}$. We used GAD67 instead of VGAT as a marker of GABAergic phenotype because the latter transporter is not expressed in POMC neurons (Stincic et al., 2018). Despite this, there is 
substantial electrophysiological evidence of synaptic GABA release from POMC neurons. Together, these data suggest one or both of the following: Gad67-expressing POMC neurons also express Vglut2, and/or there is turnover and overlap from a glutamatergic identity to a GABAergic identity in POMC neurons. Whether these phenomena represent the normal pattern of activity for this cell population, or whether they arose from a compensatory mechanism initiated from an atypical developmental program, remains to be determined.

The differences that we found in cell counts between $\mathrm{ISH}$ and IHC experiments, as well as the difference observed between the ISH studies and the GRT-PCR measures, may be due to differences in the sensitivities in the assays used. The ISH experiments on the restored mice were conducted on tissue that was collected from animals that were between nine and 13 weeks old, whereas the IHC studies and GRT-PCR were conducted on tissue that was collected from mice that were between 12 and 13 weeks old. While this age gap is not typically regarded as a critical developmental period, there may be changes occurring in the neurotransmitter landscape, which could account for the different cell counts that we measured. The means of ISH tissue preparation and probing method used to label cells can profoundly impact the sensitivity and interpretation of the assay, which may account for the varying proportions of glutamatergic POMC neurons that have been reported via ISH, let alone IHC. However, our results fit with the numbers reported previously using the same preparation and detection methods (Hentges et al., 2009; Vong et al., 2011; Dicken et al., 2012; Wittmann et al., 2013; Dennison et al., 2016). For IHC, we used three different secondary antibodies and colorimetric or fluorescent techniques to label POMC neurons. The inconsistencies in our data were not between experimental groups, but between the detection methods that were used. We found the greatest number of POMC neurons using colorimetric $\mathrm{DAB}$ as the labeling method, followed by the fluorescent markers Alexa Fluor 488 and Alexa Fluor 568, respectively. However, we also utilized two different dilutions of the POMC primary antibody, 1:1000 for the DAB and Alexa Fluor 568 sections and 1:10,000 for the Alexa Fluor 488 sections. However, given that we measured a greater number of POMC neurons with Alexa Fluor 488 than with Alexa Fluor 568, we conclude that differences that we see are more a reflection of detection subjectivity of the secondary antibodies than of the efficiency of the primary antibody used.

Overlap between GABAergic and glutamatergic cellular phenotypes and machineries is not a novel concept. Several studies have identified instances of these intersections in multiple systems, which can be influenced by cellular excitability, developmental timing, or environmental factors. For example, Kao et al. (2004) found that there is a subset of retinal bipolar cells in cats that use both glutamate and GABA, and express vesicular transporters for each molecule. Using subcellular fractionation and synaptosomal isolation, Zander et al. (2010) identified sizeable vesicle pools containing both VGLUT2 and VGAT. Furthermore, they also showed that VGAT immu- noisolates transport glutamate, and that VGLUT activity enhances the uptake of GABA and monoamines. Several studies have demonstrated that following stimulationinduced hyperexcitability of granule cells in the rat dentate gyrus, their mossy fibers can transition from a glutamatergic to a GABAergic neurotransmission and that these cells possess a GABAergic phenotype during early postnatal periods, which is suppressed in mature cells (Walker et al., 2001; Gutiérrez, 2002, 2003, 2005; Gutiérrez et al., 2003). In 2005, Gómez-Lira et al. (2005) further showed that the presence of TrkB-mediated BDNF signaling can also lead to a similar glutamatergic-to-GABAergic transition in mature cultured rat granule cells, showing that this phenomenon is not limited to a critical developmental period or supraphysiological stimulation. Ottem et al. (2004) also demonstrated that nearly all neurons in the female rat anteroventral periventricular nucleus are both GABAergic and glutamatergic and that both their excitability and VGLUT2 and VGAT vesicular pools are regulated by estradiol and photoperiodic signals, highlighting another case of external regulation of phenotypic identity. In the rat medial nucleus of the trapezoid body Gillespie et al. (2005) found GABA and glutamate co-release in the developing auditory system, which may aid in synaptic refinement and formation of the tonotopic map. More recently, Root et al. (2014) demonstrated in rats and mice that neurons from the ventral tegmental area innervating the lateral habenula (LHb), possess markers for both GABAergic and glutamatergic transmission. Furthermore, optogenetic stimulation of these cells exerts both excitatory and inhibitory influences on LHb neurons. Finally, a review by Mestikawy et al. (2011) outlines the overlap of VGLUT1, VGLUT2, and VGLUT3 with other transmitter systems throughout the central nervous system, highlighting the versatility and pervasiveness of this transporter family, both in function and anatomy. Beyond sharing neurotransmitter phenotypes, work from the Spitzer lab has highlighted complete switches from one neurotransmitter identity to another (Spitzer, 2015, 2017). In 2013, Dulcis et al. (2013) found a switch between dopamine and somatostatin neurons in response to exposure to different photoperiod lengths in adult mice, which was independent of neurogenesis or cell death. Meng et al. (2018) went on to show that in the rat paraventricular nucleus of the hypothalamus this switch occurs exclusively in dopaminergic neurons that coexpress VGLUT2 and that the switch is contingent on elevated activity in those cells.

Furthermore, coexpression of Vglut2 and Gad67 in Pomc neurons has been reported in a single-cell RNA sequencing study (Lam et al., 2017), where they observed a $24 \%$ overlap between the markers. They also reported that $87 \%$ of Pomc neurons express Gad67 and 50\% express Vglut2. The overall Vglut2 and Gad67 counts, and the triple counts seem to mostly be in agreement with the numbers that we measured in our dual- and triple-label ISH experiments, where we report more dual-labeled cells (38\%) and total Vglut2 ${ }^{+}$cells (dual-label: $56 \%$, triplelabel: $58 \%$ ), but $15-22 \%$ less total Gad $67^{+}$cells (duallabel: $65 \%$, triple-label: $72 \%)$. The directionality of the 
Vglut2 and Gad67 counts between the studies are in agreement; where, Gad67 represents a larger proportion of the Pomc population than Vglut2. Additionally, we can infer from their reported proportions that $13 \%$ of the sequenced Pomc neurons were Pomc-only, 50\% were Gad $67^{+}, 13 \%$ were Vglut2 ${ }^{+}$, and $24 \%$ were Gad $67^{+}$/ Vglut2 $^{+}$; these counts are somewhat different than our respective measures of $7.1 \%, 34.7 \%, 20.6 \%$, and $37.6 \%$. These differing counts could arise from a few factors: they used younger mice in their study, and their dataset arises from only 163 cells, which might not fully cover the entire Pomc-neuron population. Three other scRNAseq profiles of hypothalamic neurons have also identified the presence of the same four subpopulations of POMC neurons in regard to their coexpression of the two fast synaptic neurotransmitters (Campbell et al., 2017; Chen et al., 2017; Romanov et al., 2017).

An important question that can be asked regarding the restored mice arises from the lack of temporal control in the Vglut2-Cre induced, cell specific Pomc transcription. Given the widespread expression and subsequent decrease in Vglut2 expression from early postnatal development to maturation (Boulland et al., 2004; Dennison et al., 2016), VGLUT2 alone may not be the best indication of mature glutamatergic neurons. It is also possible that other differentiated neurons in the ARC may transiently express VGLUT2 during development. For the current experiments with the restored mice, we were limited to the available genetic mouse lines, and the best option was a constitutively active Vglut2-Cre driver line. Using an inducible-Cre model could potentially provide more specific insight for the role of glutamatergic POMC neurons in adult animals. However, those experiments would come with their own caveats and considerations. One such consideration is that without sufficiently early intervention the $\mathrm{FN} \Delta 2$ animals exhibit juvenile-onset obesity. An early history of excess adiposity could by itself permanently shape the hypothalamic landscape and its plasticity.

In conclusion, our triple ISH data highlight a previously overlooked phenomenon that could not be measured with previous reports using dual ISH alone. There is spatial heterogeneity between phenotypically different POMC neurons based on their markers of fast synaptic neurotransmitters along the rostral-caudal axis of the ARC. Previous work (Williams et al., 2010) demonstrated that there is an anatomical difference between leptin- and insulin-induced responses in POMC neurons. Whereas most leptin-induced hyperpolarization of POMC neurons occurred in the rostral ARC, most insulin-induced depolarization of POMC neurons occurred in the caudal portion of the ARC. Furthermore, Betley et al. (2013) demonstrated in AgRP neurons that the rostral-caudal location of the cell bodies was associated with their projection targets, where the most rostral neurons tended to project to forebrain areas, while the caudally located cells projected to hindbrain areas. Together, these findings raise questions of how overall ARC function changes developmentally and whether other ARC neuron subtypes, such as kisspeptin and dopamine neurons, also exhibit phenotypic shifts in their fast synaptic neurotransmitters.

\section{References}

Betley JN, Cao ZFH, Ritola KD, Sternson SM (2013) Parallel, redundant circuit organization for homeostatic control of feeding behavior. Cell 155:1337-1350.

Boulland J, Qureshi T, Seal RP, Rafiki A, Gundersen V, Bergersen LH, Fremeau RT, Edwards RH, Storm-Mathisen J, Chaudhry FA (2004) Expression of the vesicular glutamate transporters during development indicates the widespread corelease of multiple neurotransmitters. J Comp Neurol 480:264-280.

Bumaschny VF, Yamashita M, Casas-Cordero R, Otero-Corchón V, de Souza FSJ, Rubinstein M, Low MJ (2012) Obesity-programmed mice are rescued by early genetic intervention. J Clin Invest 122: 4203-4212.

Burke LK, Doslikova B, D’Agostino G, Greenwald-Yarnell M, Georgescu T, Chianese R, Martinez de Morentin PB, OgunnowoBada E, Cansell C, Torres LV, Garfield AS, Apergis-Schoute J, Lam DD, Speakman JR, Rubinstein M, Low MJ, Rochford JJ, Myers MG, Evans ML, Heisler LK (2016) Sex difference in physical activity, energy expenditure and obesity driven by a subpopulation of hypothalamic POMC neurons. Mol Metab 5:245-252.

Campbell JN, Macosko EZ, Fenselau H, Pers TH, Lyubetskaya A, Tenen D, Goldman M, Verstegen AMJ, Resch JM, McCarroll SA, Rosen ED, Lowell BB, Tsai LT (2017) A molecular census of arcuate hypothalamus and median eminence cell types. Nat Neurosci 20:484-496.

Chen R, Wu X, Jiang L, Zhang Y (2017) Single-cell RNA-seq reveals hypothalamic cell diversity. Cell Rep 18:3227-3241.

Chhabra KC, Adams JM, Fagel B, Lam DD, Qi N, Rubinstein M, Low MJ (2016a) Hypothalamic POMC deficiency improves glucose tolerance despite insulin resistance by increasing glycosuria. Diabetes 65:660-672.

Chhabra KC, Adams JM, Jones GL, Yamashita M, Schlapschy M, Skerra A, Rubinstein M, Low MJ (2016b) Reprogramming the body weight set point by a reciprocal interaction of hypothalamic leptin sensitivity and Pomc gene expression reverts extreme obesity. Mol Metab 5:869-881.

Dennison CS, King CM, Dicken MS, Hentges ST (2016) Agedependent changes in amino acid phenotype and the role of glutamate release from hypothalamic proopiomelanocortin neurons. J Comp Neurol 524:1222-1235.

Dicken MS, Tooker RE, Hentges ST (2012) Regulation of GABA and glutamate release from proopiomelanocortin neuron terminals in intact hypothalamic networks. J Neurosci 32:4042-4048.

Dulcis D, Jamshidi P, Leutgeb S, Spitzer NC (2013) Neurotransmitter switching in the adult brain regulates behavior. Science 340:449453.

Gillespie DC, Kim G, Kandler K (2005) Inhibitory synapses in the developing auditory system are glutamatergic. Nat Neurosci 8:332-338.

Gómez-Lira G, Lamas M, Romo-Parra H, Gutiérrez R (2005) Programmed and induced phenotype of the hippocampal granule cells. J Neurosci 25:6939-6946.

Gutiérrez R (2002) Activity-dependent expression of simultaneous glutamatergic and GABAergic neurotransmission from the mossy fibers in vitro. J Neurophysiol 87:2562-2570.

Gutiérrez R (2003) The GABAergic phenotype of the "glutamatergic" granule cells of the dentate gyrus. Prog Neurobiol 71:337-358.

Gutiérrez R (2005) The dual glutamatergic-GABAergic phenotype of hippocampal granule cells. Trends Neurosci 28:297-303.

Gutiérrez R, Romo-Parra H, Maqueda J, Vivar C, Ramirez M, Morales MA, Lamas M (2003) Plasticity of the GABAergic phenotype of the "glutamatergic" granule cells of the rat dentate gyrus. J Neurosci 23:5594-5598

Hentges ST, Nishiyama M, Overstreet LS, Stenzel-Poore M, Williams JT, Low MJ (2004) GABA release from proopiomelanocortin neurons. J Neurosci 24:1578-1583.

Hentges ST, Otero-Corchon V, Pennock RL, King CM, Low MJ (2009) Proopiomelanocortin expression in both GABA and glutamate Neurons. J Neurosci 29:13684-13690. 
Jarvie BC, Hentges ST (2012) Expression of GABAergic and glutamatergic phenotypic markers in hypothalamic proopiomelanocortin neurons. J Comp Neurol 520:3863-3876.

Kao Y, Lassová L, Bar-Yehuda T, Edwards RH, Sterling P, Vardi N (2004) Evidence that certain retinal bipolar cells use both glutamate and GABA. J Comp Neurol 478:207-218.

Lam BYH, Cimino I, Polex-Wolf J, Kohnke SN, Rimmington D, lyemere V, Heeley N, Cossetti C, Schulte R, Saraiva LR, Logan DW, Blouet C, O'Rahilly S, Coll AP, Yeo GSH (2017) Heterogeneity of hypothalamic pro-opiomelanocortin-expressing neurons revealed by single-cell RNA sequencing. Mol Metab 6:383-392.

Lam DD, Attard CA, Mercer AJ, Myers MG, Rubinstein M, Low MJ (2015a) Conditional expression of Pomc in the Lepr-positive subpopulation of POMC neurons is sufficient for normal energy homeostasis and metabolism. Endocrinology 156:1292-1302.

Lam DD, de Souza FSJ, Nasif S, Yamashita M, López-Leal R, Otero-Corchon V, Meece K, Sampath H, Mercer AJ, Wardlaw SL, Rubinstein M, Low MJ (2015b) Partially redundant enhancers cooperatively maintain mammalian Pomc Expression above a critical functional threshold. PLoS Genet 11:e1004935.

Meng D, Li H, Deisseroth K, Leutgeb S, Spitzer NC (2018) Neuronal activity regulates neurotransmitter switching in the adult brain following light-induced stress. Proc Natl Acad Sci USA 115:50645071.

Mercer AJ, Hentges ST, Meshul CK, Low MJ (2013) Unraveling the central proopiomelanocortin neural circuits. Front Neurosci 7:19.

Mercer AJ, Lentz SI, de Souza FSJ, Rubinstein M, Low MJ (2014) T-3080-OR: redefining the synaptic projection patterns of the hypothalamic proopiomelanocortin circuit. Presented at The Obesity Society Annual Meeting, Boston, November.

Mestikawy SE, Wallén-Mackenzie Å, Fortin GM, Descarries L, Trudeau LE (2011) From glutamate co-release to vesicular synergy: vesicular glutamate transporters. Nat Rev Neurosci 12:204216.

Ottem EN, Godwin JG, Krishnan S, Petersen SL (2004) Dualphenotype GABA/glutamate neurons in adult preoptic area: sexual dimorphism and function. J Neurosci 24:8097-8105.
Romanov RA, Zeisel A, Bakker J, Girach F, Hellysaz A, Tomer R, Alpár A, Mulder J, Clotman F, Keimpema E, Hsueh B, Crow AK, Martens H, Schwindling C, Calvigioni D, Bains JS, Máté Z, Szabó G, Yanagawa Y, Zhang MD, et al. (2017) Molecular interrogation of hypothalamic organization reveals distinct dopamine neuronal subtypes. Nat Neurosci 20:176-188.

Root DH, Mejias-Aponte CA, Zhang S, Wang HL, Hoffman AF, Lupica CR, Morales M (2014) Single rodent mesohabenular axons release glutamate and GABA. Nat Neurosci 17:1543-1551.

Song AJ, Palmiter RD (2018) Detecting and avoiding problems when using the Cre-lox system. Trends Genet 34:333-340.

Spitzer NC (2015) Neurotransmitter switching? No surprise. Neuron 86:1131-1144.

Spitzer NC (2017) Neurotransmitter switching in the developing and adult brain. Annu Rev Neurosci 40:1-19.

Stincic TL, Grachev P, Bosch MA, Rønnekleiv OK, Kelly MJ (2018) Estradiol drives the anorexigenic activity of proopiomelanocortin neurons in female mice. eNeuro 5.

Vong L, Ye C, Yang Z, Choi B, Chua S, Lowell BB (2011) Leptin action on GABAergic neurons prevents obesity and reduces inhibitory tone to POMC neurons. Neuron 71:142-154.

Walker MC, Ruiz A, Kullmann DM (2001) Monosynaptic GABAergic signaling from dentate to CA3 with a pharmacological and physiological profile typical of mossy fiber synapses. Neuron 29:703715.

Williams KW, Margatho LO, Lee CE, Choi M, Lee S, Scott MM, Elias CF, Elmquist JK (2010) Segregation of acute leptin and insulin effects in distinct populations of arcuate proopiomelanocortin neurons. J Neurosci 30:2472-2479.

Wittmann G, Hrabovszky E, Lechan RM (2013) Distinct glutamatergic and GABAergic subsets of hypothalamic pro-opiomelanocortin neurons revealed by in situ hybridization in male rats and mice. $J$ Comp Neurol 521:3287-3302.

Zander JF, Münster-Wandowski A, Brunk I, Pahner I, Gómez-Lira G, Heinemann U, Gutiérrez R, Laube G, Ahnert-Hilger G (2010) Synaptic and vesicular coexistence of VGLUT and VGAT in selected excitatory and inhibitory synapses. J Neurosci 30:7634-7645. 